June 2006

\title{
Retention Forces of Spherical Attachments as a Function of Implant and Matrix Angulation in Mandibular Overdentures : An In-Vitro Study
}

Sergio Manuel. Ortegon

Follow this and additional works at: https://opencommons.uconn.edu/sodm_masters

\section{Recommended Citation}

Ortegon, Sergio Manuel., "Retention Forces of Spherical Attachments as a Function of Implant and Matrix Angulation in Mandibular Overdentures : An In-Vitro Study" (2006). SoDM Masters Theses. 4.

https://opencommons.uconn.edu/sodm_masters/4 


\title{
RETETION FORCES OF SPHERICAL ATTACHMENTS AS A FUNCTION \\ OF IMPLANT AND MATRIX ANGULATION IN MANDIBULAR OVERDENTURES: AN IN-VITRO STUDY
}

\section{Sergio Manuel Ortegon}

D.D.S, Pontificia Universidad Javeriana, Bogota-Colombia

Advanced Education in General Dentistry, University of Connecticut Health Center

\author{
A Thesis \\ Submitted in Partial Fulfillment of the \\ Requirements for the Degree of \\ Master of Dental Science \\ University of Connecticut Health Center \\ 2006
}




\section{RETENTION FORCES OF SPHERICAL ATTACHMENTS AS A FUNCTION OF IMPLANT ANGULATION IN MANDIBULAR OVERDENTURES: AN IN- VITRO STUDY}

\section{Presented by}

Sergio Manuel Ortegon, D.D.S.

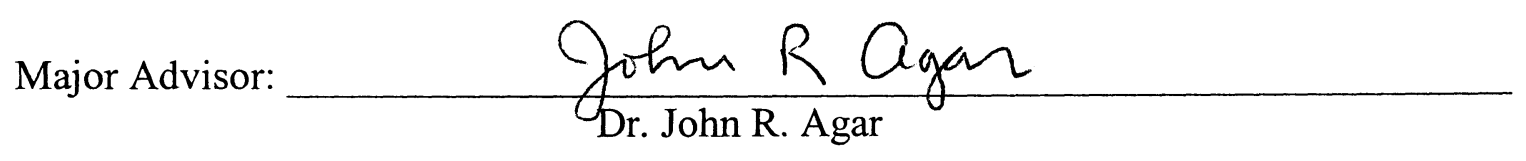

Associate Advisor: Sic ofreey $\times$ Tton psoDr. Geoffrey Thompson

Associate Advisor: Dr. Thomas D. Taylor

Associate Advisor:
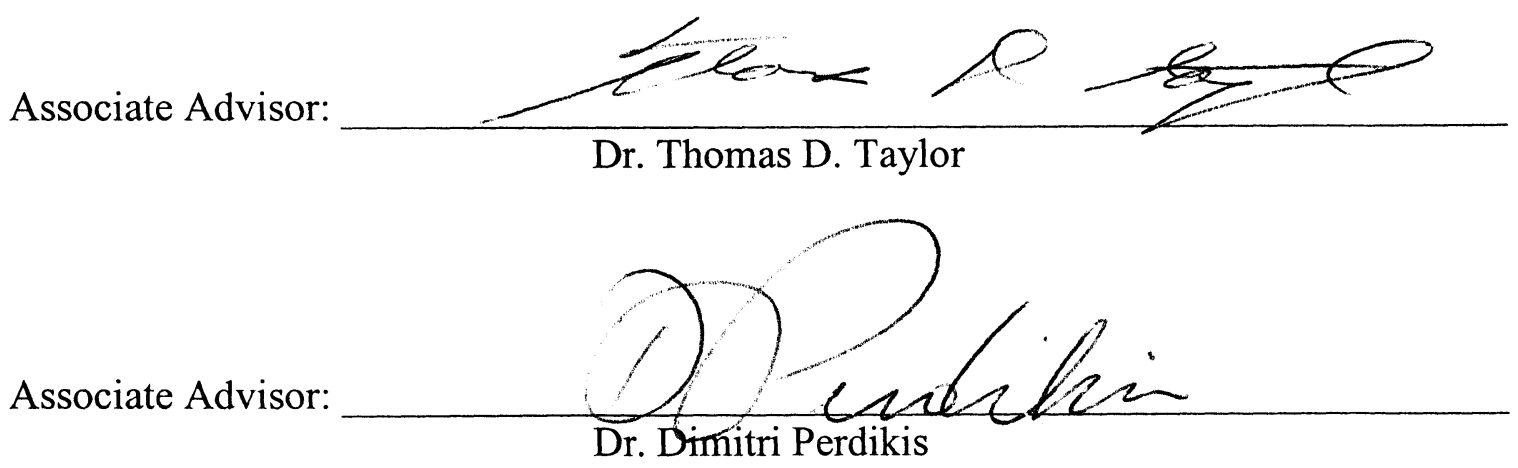

University of Connecticut Health Center

2006 


\section{ACKNOWLEDGEMENTS}

I would like to thank my research committee for taking the time to help me in achieving this endeavor.

Firstly, I would like to recognize Dr. Agar's invaluable input for this project, as well as to thank him for sharing his original ideas that served as a core for the development of this study. This project would not have been completed without his constant guidance and support.

I would like to extend my gratitude to Dr. Thompson for his dedication and mentoring throughout this process. His hard work and vast knowledge is reflected in the outcome of this project.

I would like to also thank Dr. Taylor for his motivation and advice. His support was a vital for the accomplishment of the entire project.

I would like to extend my gratitude to Dr. Perdikis who gave me constant encouragement and support in the design of the project.

My special thanks to Dr. Barndt who shared his knowledge of engineering when I needed the most.

I would not have been able to complete this project without the support and unconditional help from Eileen Chmura and Cynthia Phoenix.

Finally, I would like to recognize the help and support of all my friends, coresidents, family and above all, my wife. 


\section{DEDICATION}

To my wife Maria, and my family. 
List of Tables vii

List of Figures viii

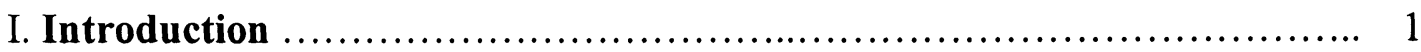

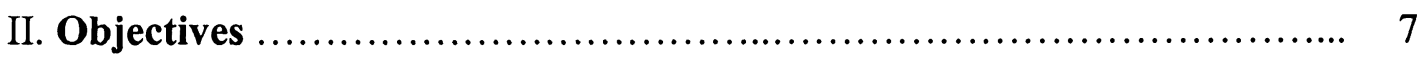

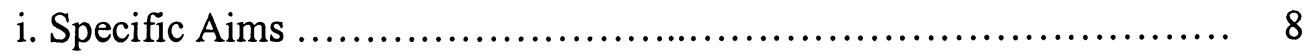

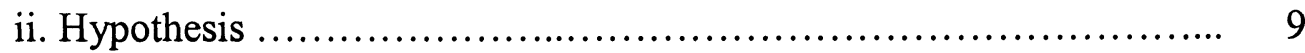

III. Literature Review ................................................. 10

1. Epidemiology of Edentulism: Current trends; need for

Mandibular Complete Dentures ............................................ 10

2. Principles of Mandibular Overdentures ................................... 12

3. Attachment development in tooth supported and retained overdentures........... 14

4. Retention and stability as confounding factors in patient satisfaction

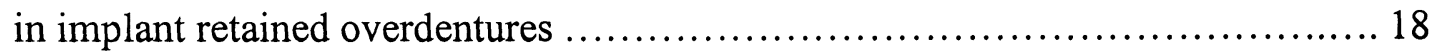

5. Rationale for attachment selection: maintenance and retention parameters...... 21

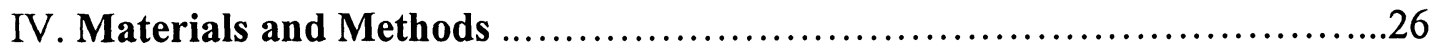

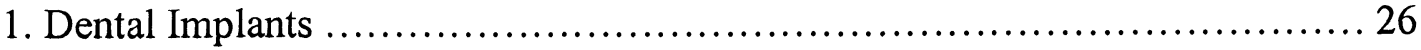

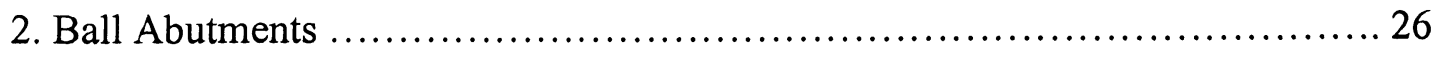

3. Ball Attachments ....................................................... 26 
3.1 Attachment characteristics measured in a Photometric Analysis.

3.2. Attachment characteristics prior and after cycling loading:

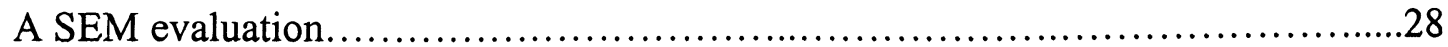

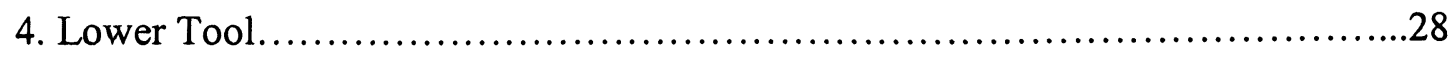

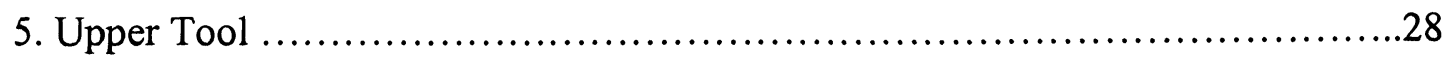

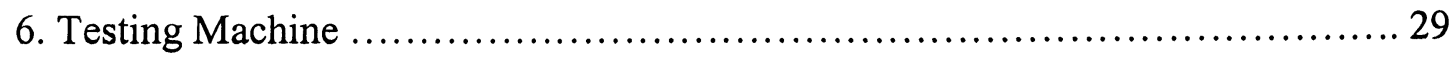

7. Data acquisition software, testing protocol................................29

8. Specimen Preparation....................................................30

8.1. Samples Description.................................................. 30

8.2. Incorporating the Attachment on the ball abutment..........................30

8.3. Incorporating the angles to the attachment................................ 30

8.4. Attachment processing................................................ 31

9. Specimen assembly for testing.......................................... 32

9.1. Changing the cycled plastic inserts for new ones...........................33

10. Statistical Analysis.................................................. 33

V. Results............................................................

VI. Discussion ......................................................... 42

VII. Conclusions ........................................................ 56

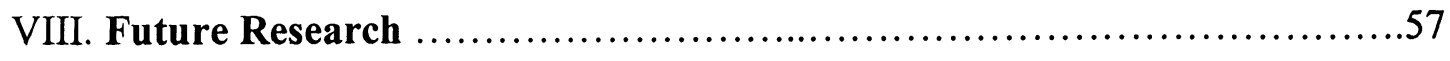

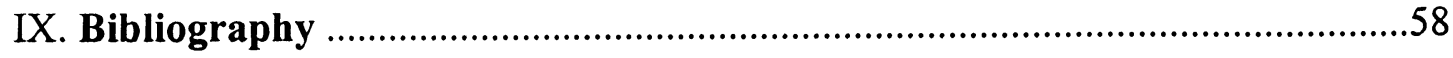




\section{LIST OF TABLES}

TABLE

I.

TITLE

PAGE

This table shows one of the 60 Excel spread-sheets used to

Tabulate the MTS data. (0-0 Specimen, 1F)
.68 



\section{LIST OF FIGURES}

FIGURE

TITLE

PAGE

Fig. 1. Dalbo series: Gold lamella and housing. PVC sleeve. No rotational capability over the abutment. Preiskel. Overdentures made easy........................................................ 69

Fig. 2. Astra Implant. 4.0 X 13mm...................................... 69

Fig. 3. 2.25mm diameter, 1.5mm height, Astra Ball Abutment............. 63

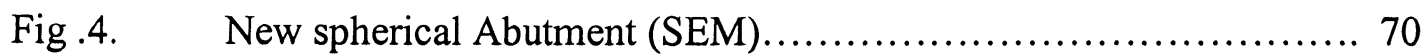

Fig. 5. Ball abutment after cycling ..................................... 71

Fig. 6. Preci-Clix female attachment (Housing and Insert).................71

Fig 7. Plastic Insert................................................... 71

Fig. 8. Rotational capability threshold of Preci-Clix attachment on Astra implant. 12.8 degrees......................................72

Fig. 9. Diameter of new plastic insert..................................73

Fig. 10. SEM view of randomly selected new attachments showed

Fabrication discrepancies.........................................74

Fig. 11. Figure depicts a SEM image of an attachment tested on

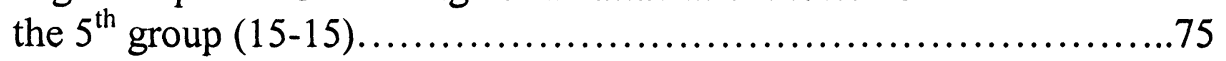

Fig. 12. Professional Vise (Mandible analog)..............................76

Fig. 13. Aluminum angled blocks ( $15^{\circ}$ pair).....................................................76

Fig. 14. Upper aluminum Tool..........................................77

Fig. 15. Instron 5581 series, Norwood, MA.............................78

Fig. 16. Test Works 4®, version 4.08 B Build 868. 1995-2003 MTS systems corporation. 
Fig. 17. Implant-Attachment Configurations.............................. 80

Fig. 18. Attachment insertion and fixation prior processing ...................81

Fig. 19. Aluminum blocks being used on a vise to direct angulation of the abutments and attachments which in turn run perpendicular to their blocks slope. The figure shows a partially opened vise with 10 degree aluminum blocks...................................81

Fig. 20. Attachment processing. Figure shows the upper aluminum jig being held into position prior injecting the acrylic resin. 82

Fig. 21. Attachment processing. Figure depicts the appearance of the Acrylic after setting and removing the top half of the Upper Aluminum Tool, Grip has been raised....

Fig. 22. Upper and Lower Jigs denoting the $0^{\circ}$ attachment insertion to $15^{\circ}$ implants.

Fig. 23. Upper and lower tools holding parallel implants and parallel Attachments

Fig. 24. Complete set up on Instron machine..............................86

Fig. 25. Configurations 4 and 5. Attachments processed following Non-parallel implants long axis. . .86

Fig. 26. Arrow depicts plastic permanent deformation of the 15-15 sample......87

Fig. 27. Arrow depicts the contra-lateral side of one cycled 15-15 Plastic insert. Note the lack of changes. .88

Fig. 28. Diagram depicts the 15-0 configuration, internal and external Aspects are labeled.

Fig. 29. SEM (15-0). Red arrow depicts internal aspect, yellow indicates External. See the difference in wear patterns........................90

Fig 30. Arrow depicts plastic disintegration of the 15-0 configuration.........91 


\section{INTRODUCTION}

According to the Third National Health and Nutrition Examination Survey (NHANES), edentulism has been decreasing by $10 \%$ every decade. Even with this significant percentage decline, an increase in the actual number of adults will lead to an increase in the number of complete dentures needed over the next two decades. A recent study from the Center for Disease Control (CDC) that used data from the Behavioral Risk Factor Surveillance System (BRFSS) confirms a decline in edentulism of the population over 65 years old. The authors did not account for the steady increase in the number people who are rapidly falling into this age bracket; nor had they included institutionalized population or elderly without residential telephone service. Other epidemiological studies have argued that although the edentulous population is decreasing the need for complete dentures will remain the same because of the increase in size and age of the older population. Complete dentures are and will continue to be needed for edentulous patients.

Traditionally in clinical scenarios where there has been extreme mandibular bone atrophy, the fabrication of complete dentures represents a challenge to the restorative dentist. Retention can not be achieved as in the maxillary arch. Mandibular edentulous ridges could provide adequate stability and support for conventional complete dentures in patients where minimum resorption is evidenced. On the other hand when there is lack of ridge height, width and extreme bone resorption is observed, teeth and/or endosseous dental implants could be used to achieve appropriate denture stability and retention. 
The dental literature describes many mechanical advantages and disadvantages that teeth have provided to mandibular overdentures. The disadvantages include caries and periodontal disease. Additionally, the cost of having to perform endodontic and restorative treatment on terminal overdenture abutments was at times a significant investment that did not have a good long term prognosis. Natural tooth supported overdentures could inadvertently cause mechanical disadvantages when the coronal portion of the abutment teeth that contacted the intaglio surface of the overdenture prevented complete seating of the prosthesis, generating fulcrum lines and the potential for rocking and instability. As early as the late eighteen hundreds, attachments connecting the abutment teeth to the prosthesis to augment retention were reported in the dental literature.

Osseointegrated implants were initially used to support fixed prosthesis in edentulous patients who experienced problems with mandibular complete dentures. Later, they were used for single or multiple fixed implant supported restorations in partially edentulous patients and subsequently to provide mechanical attachment for support and retention of overdentures.

Clinical studies have shown that dental implants used in mandibular overdenture retention contribute to the preservation of residual ridges, improve masticatory function and increases patient satisfaction. This restorative treatment has demonstrated to be a cost- effective option when compared to more expensive fixed reconstructions requiring more implants, components and technique sensitive laboratory support. 
Some studies and expert group consensus have stated that two implant supported mandibular overdentures should be considered as the standard of care for edentulous patients. Methods of attachment retention have evolved following the principles and experiences with tooth born overdentures. Dalla-Bona attachments, bar and clip systems, telescopic crowns and magnets were adopted by the implant supported discipline. Studies comparing stud, bar and clip and magnets were done invitro and in-vivo in an effort to analyze which attachment provided the most retention, which provided the most stability and which produced higher or lesser forces on the implants and finally which gave patients the most satisfaction.

Globalization of this prosthodontic treatment modality and manufacturing variables with different components, affects decisions during treatment planning; which include; attachment selection, durability and maintenance frequency of some of the components.

A direct relationship has been observed between retention, stability and patient satisfaction. Research has demonstrated that magnets are the least retentive of all.

Balls were the first abutments adopted form tooth born overdentures, and these were used in external hexed implants. One of the major prosthetic complications that practitioners faced was screw loosening. With the evolution of dental implants to internal connection, the issue of ball abutment loosening decreased considerably. Presently the use of bar and clips are primarily restricted to specific treatments such as implants with extreme divergence. Bars are expensive, technically sensitive; they require more space and initially more clinical time. Clinical reports 
and technique articles have discussed the potential for rotational capability of ballattachments and advocate their use in non-parallel implant situations that are not too extreme.

Spherical attachments have been studied relative to retention in static pull tests. Most of these in-vitro studies simulate two implant retained overdenture models where implants are placed parallel to each other and perpendicular to the path of insertion of the overdenture analog. One study attempted to evaluate the influence of the implant angulation variable in a one implant overdenture model. There is no research data to date, that studies the two implant overdenture model, that shows how attachments and ball abutments behave in terms of retention when they are subjected to clinical scenarios in which the implants are placed in divergent angulations from each other and attachments are processed following the non-parallel implantabutment configuration or when attachments are placed parallel to each other in an attempt to correct the misaligned implant configuration.

The primary aim of this study is to evaluate the rotational corrective capability of the Preci-clix attachment system on Astra-Tech spherical abutments in terms of retentive values in diverse angle permutations. The Preci-clix attachment will also be evaluated in non-parallel implant configurations when the attachments are processed following the implant-abutment angulation.

The in-vitro testing model has two components; a lower element that simulated a mandible capable of predictably retaining two implants parallel to each other or divergent from each other at $10^{\circ}$ or $15^{\circ}$; and an overdenture analog 
consisting of a split aluminum cast that holds the attachments in place at $0^{\circ}, 10^{\circ}$ or $15^{\circ}$ from the vertical reference plane.

One of the objectives in the design of the testing model was to be able to fabricate the samples in the identical testing replica where they were going to be tested. In an attempt to minimize operator variability all samples were fabricated by one operator. The same materials and methods were followed for each of the specimens. There where 5 main groups or configurations and 6 specimens per group. A total of 30 specimens were evaluated.

The Preci-clix spherical attachment (Yellow plastic, medium retention) using Astra-Tech implant abutments were tested in the following coupling situations: Sample \#1: parallel implant abutments-parallel attachments; Sample \#2: $10^{\circ}$ divergent implant abutment-parallel attachments; Sample \#3: 15 divergent implant abutment-parallel attachments; Sample \#4: $10^{\circ}$ divergent implant abutments- $10^{\circ}$ divergent attachment and Sample \#5: $15^{\circ}$ divergent abutment implant-15 divergent attachment.

It was determined that a one pull test did not reflect a true clinical scenario because an overdenture is usually subjected to multiple insertions and removals. Cyclic loading simulating three years of use at a cross-head speed of $50 \mathrm{~mm} / \mathrm{sec}$ was performed.

The samples were evaluated in an Instron Machine Model 5581, and the data acquisition was performed with the Test Works software from the MTS Company.

Analysis of Variables (ANOVA) and Scheffe's post-hoc analyses were used to determine statistical significance between retention mean loads of the groups. No 
statistical significance was found in retention mean load of group \#1 and \#2. Higher initial retentive values were observed in group \#5. Although there are other variables involved in attachment wear in-vivo, this in-vitro study suggests that implants with a combined divergence within the $20^{\circ}$ range could be handled predictably with parallel attachments. Moreover it confirms that attachments processed following implant divergence in a combined excess of 30 degrees would yield initial higher retention values with a tendency of decrease over time.

This research study provides evidence of the rotational corrective capability of spherical attachments and predicts a threshold of compensation for implant-abutment non-parallelism. It also offers new evidence of what happens to retention values when Preci-Clix attachments are processed following non-parallel implants. Based on these observations, suggestions in terms of maintenance will be made. These original materials and methods designs could be used in future research to evaluate different attachment-implant systems with diverse angulations. 


\section{OBJECTIVES}

When inadequate retention and stability are evidenced due to insufficient alveolar ridge relationships, neuromuscular coordination and inadequate vestibular depth, the prescription of mandibular implant-retained overdentures should be considered whenever possible. Several retentive systems have been developed and specific applications for these have been advocated. The bar-clip system historically has been utilized in non-parallel two implant retained overdentures. Magnets, according to research yield low retentive values and are affected by the presence of plaque and saliva. Moreover, authors have reported in technique articles, the properties of ball abutments; they recognize that these have an inherent rotational capability that could be applied up to a point in non-parallel implant situations. Research has evaluated breakaway forces of stud attachments under arbitrary or controlled speeds, in vitro, in vivo, in cross-over studies, in static loads and in cyclic loading. Most studies, evaluated parallel implants with parallel attachments to a vertical reference plane, which is a desirable clinical situation; however in many clinical scenarios implants are placed in a non-parallel orientation for multiple reasons such as; surgical expertise, anatomical restrictions and planning deficiencies.

Furthermore there are few case report articles and only one laboratory based research study that comments on how to deal from the prosthetic point of view with divergent implants using ball attachments. A small number of technique and review articles recognize the potential maintenance issues when dealing with non-parallel implants where the attachments are processed mistakenly following the implantabutment angulation. Some agree that the attachment system would be forced into 
place and that wear in the components is expected to occur. So far no one has evaluated these variables, in a two implant overdenture model and how they affect retention throughout time.

The primary objective of this study was to evaluate whether divergent implants could be predictably managed in terms of retention with attachments placed parallel to the path of withdrawal, when compared to a parallel implant scenario. A related objective was to evaluate the attachments behavior in terms of retention when processed following a divergent implant overdenture situation.

\section{i. Specific Aims}

1. This study developed a two implant retained overdenture research model suitable for the evaluation of spherical attachment retention in parallel and nonparallel implant situations. The results of this investigation will evaluate the use of the rotational corrective capability of Preci-Clix attachments in clinical approaches to correct non-parallel implant scenarios and at the same time will investigate angulation correction threshold for the Preci-clix attachment used on Astra implants.

2. This research model allowed varying the attachments' angulation to correct or to follow predetermined implant angulations, with the objective of replicating clinical scenarios where the attachments are processed regardless of the implants degree of parallelism. The significance of these results will suggest whether attachments that are processed following a divergent implant configuration have the 
potential for a quick drop in retention, which in turn would yield a high incidence of maintenance and decreased predictability for attachment durability.

\section{ii. Hypothesis}

The null hypotheses tested were:

-There is no difference in retention loads between the first group $\left(0^{\circ}\right.$ implants$0^{\circ}$ attachments $)$ and the second group $\left(10^{\circ}\right.$ implants $-0^{\circ}$ attachments $)$

-There is no difference in retention loads between the first group $\left(0^{\circ}\right.$ implants$0^{\circ}$ attachments) and the third group ( $\left(15^{\circ}\right.$ implants- $0^{\circ}$ attachments).

-There is no difference in retention loads between the first group $\left(0^{\circ}\right.$ implants$0^{\circ}$ attachments) and the fifth group ( $15^{\circ}$ implants- $15^{\circ}$ attachments). 


\section{LITERATURE REVIEW}

\section{Epidemiology of Edentulism: Current trends, need for Mandibular Complete Dentures.}

Recent Epidemiological reports from the Center for Disease Control (CDC) show that the percentage of older adults who have retained their teeth has increased gradually. [1] This trend is expected to prevail based on public health policies such as water fluoridation and improvement in dental care accessibility. [2] Although studies confirm a decrease in edentulism, its prevalence in the adult population is still high: 10\%; as reported by the National Institute of Dental and Craniofacial Research.[3]

A Behavioral Risk Factor Surveillance System (BRFSS) survey was used to measure the percentage of edentulous people over the age of 65 years. The study included 50,635 participants; but did not incorporate institutionalized elderly, nor people without residential telephone services. This survey found huge discrepancies between states; the prevalence of edentate persons ranged form $13 \%$ in California to $42 \%$ in Kentucky. [1] Another study by the CDC comparing two different surveys; one from 1988-1994 and the other from 1999-2002; indicated that the prevalence of tooth loss continues to decline. This study provides further evidence that previously adopted oral health measures have been working properly.[4]

However, a 1988 study noted that regardless of the decline in edentulism, the demand for complete dentures would actually increase because of the aging trends in the population.[5] 
Some authors have shown that epidemiologic literature has historically drawn conclusions on trends in edentulism without taking into consideration the growth of the older population.[6, 7] Estimates based on national epidemiologic survey data indicates that edentulism has declined by $10 \%$ every decade, however the adult population in need of 1 or 2 complete dentures is expected to increase from 33.6 million adults in 1991 to 37.9 million adults in 2020.[7]

Through media, education, availability of dental insurance and financial stability the edentulous population has become more aware of a variety of oral health resources for the treatment of their condition. Research and clinical experience have shown that edentulism is a condition that could affect oral, general health as well as overall quality of life. $[8,9]$

The presence of removable prostheses in the edentulous population influences the ease of chewing and the pleasure resulting from experiencing different foods. Studies have shown a correlation between the ability to eat food and nutrition intake. Edentulous individuals living independently have been shown to have a lower nutrient ingestion compared to dentate adults.[10,11]

Historically mandibular complete denture success has been linked to several factors such as patient psychological acceptance, ridge height and width, muscle attachment location, availability of masticatory mucosa, opposing dentition, and occlusal stability. [12]

Edentulous patients with severely resorbed ridges often report having negative experiences with their conventional dentures because of an impaired load-bearing capacity.[13, 14] Clinical research supports clinical observations where complete 
denture wearers' mandibular bone is lost four times more, compared to the maxillary bone.[15, 16] This change in the edentulous anatomy makes the fabrication of mandibular complete dentures cumbersome. In an often cited article, Atwood[17] described the continuous reduction of residual ridges in complete denture wearers as a "major oral disease entity". Edentulous patients who suffer from the results of residual bone resorption and experience "maladaptive" characteristics with their mandibular dentures will benefit from implant supported-retained prostheses.[18, 19]

\section{Principles of Mandibular Overdentures}

Elderly new dentures wearers have a bad prognosis for learning new muscular patterns. Postponing edentulousness to an advanced age has been said to cause maladaptive behaviors in patients and problems for the dentist.[20] Nevertheless European practitioners began recognizing by the $19^{\text {th }}$ century that tooth supported overdentures were an option for patients that physiologically could not deal with the loss of all their teeth. In 1856 Ledger described a prosthesis that resembled an overdenture.[21] By the 1960's there was enough empirical information to consider the concept of tooth supported overdentures as a viable predictable treatment modality.[16] Moreover, authors hypothesized that tooth supported overdentures had characteristics that made them superior to conventional complete dentures due to improved propioception, ridge preservation, more stability and preservation of bone.[22-24] Miller advocated overdentures as means of preserving residual alveolar bone, and he reported a reduction in bone resorption over a period of 6 years in 46 
overdenture patients.[23] A classic article from Crum and Rooney [25] confirmed previous findings in a study composed of two cohorts of 8 patients each; the $1^{\text {st }}$ group; had a maxillary conventional complete denture opposing a mandibular overdenture resting in two endodontically treated canines, and group 2; had conventional maxillary and mandibular complete dentures. Their results suggested that the use of mandibular overdenture helps preserve alveolar bone in the mandible.

Several articles speculated on the role of propioception in the patient treated with overdentures. [26, 27] Pacer and Bowman [28] studied the perception of occlusal loads in overdenture patients. They found that in load levels of above $2 \mathrm{~kg}$, the overdenture patients were able to discriminate loads better than patients with complete dentures. They figured that this was due to the fact that tooth supported overdenture patients had more discriminatory ability because of proprioceptive features of the remaining teeth.

The land-mark articles that described simplified overdenture treatments were published in 1969 by Morrow et al[29] and by Lord and Teel[30]. Although it seemed to be a predictable prosthesis because it provided extended support and prevented bone resorption, it added to the treatment cost because it involved elective endodontics. The endodontic treatment allowed for a shortened dental crown, which created adequate space for the overlying artificial denture tooth and denture base.[16] Longitudinal studies evaluating long term prognosis of this treatment modality were initiated. In one study 44 overdenture patients were followed up for 5 years; the authors found that caries percentages ranged form $2.1 \%$ to $21 \%$, and that $94 \%$ of the abutment teeth needed periodontal treatment. They concluded that professional oral 
maintenance is a must and that it has to be performed at least once a year.[31] Toolson and Taylor [32] in a ten year study that included 89 patients found that most of the abutments have progressively lost attached tissue, and that patients run the risk of developing caries and losing their abutment teeth unless they maintained excellent home care with fluoride solutions plus professional care.

Overdentures supported by natural teeth were considered a gold standard in the 70's and 80 's. Patients could chew better, their ridges did not resorb as quickly and their dentures were more stable and retentive.[16]

Retentiveness was a feature that was added to the overdenture concept with the intention of returning the patient to as nearly normal function as possible. The use of attachments in combination with standard complete denture principles has been shown to improve both retention and stability of overdentures.[33]

\section{Attachment Development in Tooth supported and Retained Overdentures}

The concept of attachment stabilization and retention for tooth supported overdentures originated in Switzerland around 1898.[34] Preiskel [21] described retention as the force that resists withdrawal along the path of insertion, and argued that attachments help with retention and also aid in occlusal support and stabilization. Brewer and Morrow[35] described different attachments systems; such as; telescopic crowns, studs, bars and magnets, used in overdentures. By 1973 Mensor[36] organized attachments in a compendium known as the EM attachment selector. It 
was a color coded system that had information for more than 105 different attachment systems.

Brewer [35] classified attachments according to shape, design and primary area of use; the main category was determined by its location in respect to the clinical crown: and included intracoronal and extracoronal attachments. The radicular classification was based on design, and included the most popular attachments: telescope, stud and bar attachments.

Preliminary studies tended to evaluate any deleterious effect on the abutment teeth.[34] In 1956, Fenner and Gerber[37] used models to evaluate the forces produced by two different attachment systems. They found that there was no tipping of the abutment teeth with cylindrical attachments when compared with ball-type attachments.

A ball attachment was described either as an extraradicular or as an intraradicular attachment. The extraradicular one has the male element projecting from the root surface of the preparation and the female on the intaglio side of the prosthesis.[21] Some examples of stud attachments used in tooth retained overdentures are: Microflix, Ceka, Rhein, Rotherman, Bredent, Dalla Bona (Figure 1) and Gerber.[35]

Stud and Bar attachment studies recognized the need for a stress breaker incorporated within the attachment system to avoid torque on the abutment teeth and to simulate tissue resiliency.[37] Brewer [35] on the other hand, assured that in a well developed denture base with careful positioning of the attachments, the need for a resilient system becomes questionable. 
Early studies of the Bona-Ball anchor showed that after 50,000 insertions, the retention was reduced about $20 \%$. The author stated that the retention could be reestablished by bending the female lamella inwards.[38] This attachment was resilient and rotated upon a stud. Its nonresilient analog: the Bona-Cylinder anchor; had no rotation and had the potential to cause torque.[35] The Rothermann attachment was introduced in 1972 and had a lot of popularity due to its low height. [35]

Stud attachment designs although used for decades were seldom evaluated. Information in terms of retention was usually provided by the manufacturer.[39] It was not until Lehmann and Armin in 1978[40], that attention was paid to this feature. In this study the breakaway forces of stud attachments under arbitrary speeds were tested. In 1985 Steward and Edwards[41] also evaluated retention and cautioned against direct extrapolation of the results to clinical situations.

Laird and Grant[42] evaluated retention forces in both ball and magnets. They displayed graphic recordings of retention force versus distance of separation and noted the resistance of stud attachments to reseating when compared with magnets.

Early studies performed by Leung and Preiskel[39] examined the relationship between retention values and morphology of the male portion of various models of stud attachments. They found that parallel sided studs had the longest retentive distances and those relying on the ring-shaped springs engaging depressions on the sides of the male portion had the shortest. 
Bars units were selected on the basis of space available, the shape and the curvature of the ridge and the type of the defect to be replaced. Authors recognized a greater need for space when compared with stud attachments. Furthermore, they acknowledged the fact that the bar superstructure made the acrylic prosthesis weaker when compared to complete dentures without bars. Initial bar designs could be divided in two groups; those that allow for slight movement between the attachment components and rigid bars. Bar attachments' resiliency depended on the shape of the male component, thus squared-shaped are rigid and round or pear-shaped were resilient.[21]

Attachments groups often were named after their developers or manufacturers; that is the case of the Dolder bar joint. Dr. Dolder at the University of Zurich, in a follow up on 800 patients restored with bar retained overdentures concluded that this retentive mechanism was very successful.[43] Authors begun evaluating bars and studs. Early comparisons concentrated on the effects of splinting or not splinting overdenture abutments. Studies recognized that stud attachments allowed for independent tooth movement, meanwhile the bar prosthesis because of its splinting mechanism was more likely to cause movement as a single unit, under functional load.[44]

In a bar design two main concepts were available; the first was the Dolder bar[43] that consisted of a male and female rider; both made from gold, the second type was the Hader bar, which consisted of a gold bar and a plastic rider or female.

Mensor, who was considered an authority on tooth retained overdentures in the 70's, stated that splinting with a bar system had advantages over splinting with 
stud-type attachments that outweighed its inherent disadvantages of bulk.[44] On the other hand Preiskel[21] recognized that bar systems had to be used cautiously due to the bulk of the bar and related structures. In addition to a tridimensional space evaluation, plaque accumulation was a concern.

In terms of treatment planning for overdentures, its principles were developed early on; interoclusal space and basic mechanical principles were a concern; for instance authors believed that teeth should be treated endodontically to allow for maximum crown reduction and improved crown to root ratios and that crowns should be reduced to the level of the residual ridge in order to permit space for both the attachment and overlying tooth.[16, 44]

Overdenture qualities such as retention and maintenance were acknowledged mainly in review articles, which referenced concepts and empirism. Studies about tooth supported overdentures drifted towards implant supported and retained overdentures when osseointegrated implants became available.[45]

\section{Retention and stability as confounding factors in patient satisfaction in implant retained overdentures}

The earliest reports on implant retained overdentures come from Bodine in 1974[46] in which he reported a $52 \%$ survival rate over a 15 to 22 year period. He used subperiosteal implants. When osseointegrated implants became available interest in implant survival was the topic of interest. The implant system developed by P.I. Branemark[47] was originally described to be used in screw-retained full-arch fixed 
prosthesis. Later one its use reached patients with maladaptive behaviors with mandibular complete dentures. Once osseointegration became a predictable procedure, the prescription of dental implants for patients who suffered from inappropriate mandibular anatomy became popular. [48] A 15 year study by Adell and collaborators confirmed that dental implants could provide predictable, high level, long term success rates.

Implant development transitioned from fixed applications to provisionalization procedures between stage II surgery and insertion of the final prosthesis.[49] As multiple implant systems developed, the attachment companies were driven to devise their components for compatibility with major implant companies. Since implant predictability has been established; interest on maintenance and patient satisfaction with the implant retained overdenture surfaced.[50]

Retention was appreciated as an important factor in patient satisfaction assessment in conventional complete dentures.[51] Authors in the Netherlands, in a randomized multicenter study, evaluated patient satisfaction with mandibular conventional complete dentures, mandibular conventional complete dentures combined with vestibuloplasty and with overdentures supported by two implants. They found that implant supported mandibular overdentures consistently provided higher general satisfaction scores compared to both alternative treatments.[52]

Furthermore, in a randomized clinical trial with 151 patients that involved a comparison of conventional mandibular dentures and bar and clip retained mandibular overdentures, Geertman et al[53] found that patients with implant 
overdentures had higher general satisfaction ratings than patients with conventional dentures.

Research comparing implant supported fixed prostheses and implant supported mandibular overdentures suggest that the later could be considered as a viable alternative in terms of patient overall satisfaction. Such qualities were observed in a cross-over study by Feine et al[54]. These authors found that $50 \%$ of the patients that chose the removable design for ease of cleaning and esthetics.

Other authors compared the number of implants used, as well as, the type of attachments used in terms of patient satisfaction. Wismeijer et al[55] in a retrospective study, evaluated patient satisfaction reports with mandibular overdenture treatments either with 2 implants with ball attachments and/or 2 implants with a bar and/or 4 interconnected implants. No significant differences were found, and most of the subjects expressed satisfaction.

As retention and stability were considered two premier qualities in implant supported mandibular overdentures; research studies were developed in turn, to evaluate the interaction of the different attachment systems and clinical outcome in terms of patient satisfaction and burden of maintenance.[56]

In terms of stress being exerted on the implants, the bar and clip system has been said to cause more bending moments in finite element analysis studies.[57] Studs attachments are less costly, less technique sensitive, and easier to clean[58, 59] than bars. Furthermore reports on hyperplasia are more common on the bar modality.[60] 
Studies have shown that there is a direct relationship between retention, stability and patient satisfaction. $[54,58]$

\section{Rationale for attachment selection: maintenance and retention parameters}

There is considerable amount of literature that analyzes attachment systems of implant-retained overdentures in relation to retention, patient satisfaction and maintenance.

The use of a bar supported overdenture is favored by some clinicians, $[61,62]$ while others prefer free standing attachments. Studies are mixed or have not found significant clinical advantages for bars or free standing attachments. [63] The choice should be based on a particular clinical need. However, frequently the choice relates to a clinician's familiarity with a particular type of attachment and implant system or dental laboratory. Bar and clip retained and supported overdentures require more restorative space, are initially more expensive and technically more complex than free standing attachment systems. [56, 63]

However authors have stated that spherical attachments cannot be used when implant placement is not parallel.[61, 62, 64] Nevertheless, only a few articles outlined that many spherical attachment system designs can function appropriately when implants lack parallelism if the female attachments in the prosthesis are positioned parallel to each other and to the path of withdrawal of the prosthesis. [65, 66] 
Wiemeyer et al,[66] in a preliminary study concluded that when implants placed for a mandibular overdenture are not parallel to each other, some spherical attachment systems can allow predictable retention. They observed that stud attachments that are to move freely on a sphere, can be made parallel by adjusting the position of the matrixes to the path of removal of the overdenture. In addition the authors suggested that complete seating and predictable retention was possible even when non-parallel implants varied as much as $60^{\circ}$ from each other, for a particular gold attachment. Although these observations were done on Straumann ball abutments with a particular gold attachment, the concept applies to other spherical attachment where the matrices can rotate on the patrix.

On the other hand, the rotational capability of the spherical attachments is limited as to how long their lamella is over the stud circumference[65] and how thick is the neck of the stem connecting the ball abutment. In a research study by Gulizio et al[65] matrices demonstrated a smaller range of rotational capability.

Longitudinal clinical studies on maintenance of the implant retained mandibular overdentures in terms of the retentive components have yielded controversial results. Some authors have claimed that spherical attachments have more maintenance issues when compared to bar and clip systems.[67]

Walton,[67] in a 3 year follow up study on 87 subjects compared the bar and clip system versus the Ti Matrix and found that the Ball attachment was significantly more likely to require patrix tightening or matrix replacement, while the bar was more likely to require activation of the matrix. She also observed the high incidence of c- 
spring fractures in the Ti matrix system. Other studies also outlined the flaws of the design of the Ti Matix.[68]

In an earlier study Walton et al[69] comparing bars and ball Titanium attachments, found that the later needed considerably more adjustments and concluded that bars should be used instead of this particular ball attachment she studied.

On the other hand, there is more compelling clinical evidence that if a different type of ball attachment is used on implants other than external hexed systems; bar and clip attachments will need more maintenance. For instance in a randomized prospective 5 year prospective study, Gotfredsen et al[70] found that during the first year of function, significantly more complications/repairs were registered with the bar group than with the ball group.

The clinician should gather the available evidence in terms of maintenance, cost, ease of fabrication and clinical situation before making decisions about what attachment should be used.[56]

The trend in ball and ERA-Locator type attachments has changed from having to drill out the whole female component from the intaglio of the overdenture once it loses retention; [14] to having to exchange the acrylic insert within the female housing without removing the entire female component from the denture.

Literature on retention of attachments in implant-retained overdentures can aid the clinician in attachment selection based on the amount of retention desired rather than anecdotal findings.[71] 
Stud attachments provide varying degrees of resiliency in both vertical and horizontal directions. Magnetic attachments do not posses vertical resiliency or lateral stability.[39] Its use could be indicated in elderly patients, who require attachment systems that permit ease of prosthesis placement and removal.[72]

Walton et al[73] simulated 3 years of insertion and removal of a bar-clip assembly, and compared differences in retention between metal and plastic clips; the study found that greater wear was observed on the Gold bar when metal clips were used. In a later study Gamborena, et al [74]tested ERA attachment retention variability in a fatigue loading model and found that after 5,500 cycles there was no difference in retentive values between the four ERA colored coded attachments. Shortly after, Setz, et al[75] evaluated wear and retentive forces of commercially available attachments: studs, bars and magnets of 4 implant systems. This study performed cyclic loading that simulated approximately 5 years of use. They found that different attachments show a wide variety of retention; ranging from $3 \mathrm{~N}$ initial retention of magnets to $85 \mathrm{~N}$ initial retention of titanium matrices. They found that some systems increased their retentive values after 1,500 cycles (Gold Matrix, Dolder bar)

Other authors developed similar in-vitro and in vivo studies evaluating retention characteristics of attachment systems for overdentures. For instance Chung et al[76] performed an in-vitro evaluation in a one pull test of 9 different attachment systems; they concluded that the ERA (grey) had high retention; Locator (white LR), Spheroflex ball, Hader Bar \& metal clip, ERA (white) medium retention; the Locator 
(pink LR) low retention; and the Shiner magnet had very low retention. However they concluded that the nature of the oral environment is difficult to duplicate in vitro.

Petropoulos and Smith[77] compared two stud diameters, ERA and ZAAG attachments in terms of retention. Although this was a single pull study they suggested the use of the ZAAG attachment in cases where high retention was needed, and extra stability was required. The standard ball attachment with a diameter of $2.25 \mathrm{~mm}$ was recommended for severely resorbed mandibles and its average load was of $17.8 \mathrm{~N}$.

An in-vivo study by Van Kampen et al[78] evaluated 3 different attachment systems in terms of retention at baseline and after 3 months of use. The tested attachments were magnets, bar-clips and studs. Maintenance was recorded as well. The mean retention forces for magnets, bar and clips and ball attachments were 8.1, 31.3 and $29.7 \mathrm{~N}$ respectively. This was a one pull study which concluded that bars and balls have higher retentive values and low maintenance issues in the 3 month evaluation.

Retentive studies have followed empirical observations when deciding factors such as; speed of overdenture removal,[79] and frequency of removal[73]. Botega et al[80] used Sarnat's study speed of $50 \mathrm{~mm} / \mathrm{min}$ and Walton's study frequency of removal of 5 times per day, when evaluating studs and bar-clip systems. The study concluded that both attachment systems showed satisfactory results before and after cycling. 


\section{Materials and Methods}

With the aid of the department of Bioengineering of the University of Connecticut Health Center a device that simulated a mandible and an overdenture was designed and constructed. It had the capability for adjusting implant and attachment angulation. The instrument consisted of the following components:

\section{Dental Implants}

Two Astra-Tech Osseospeed $4.0 \times 13 \mathrm{~mm}$ implants were screwed into prefabricated holes made into two $8.5 \mathrm{~mm}$ X $8.5 \mathrm{~mm}$ X $15 \mathrm{~mm}$ long machined epoxy resin-glass fiber composite blocks, (G-10, Piedmont Plastics), in a perpendicular relation of their flat base. (Figure 2)

The G-10 is said to have a similar modulus of elasticity to that of bone, this material's Elastic modulus is $\mathrm{E}=18.6 \mathrm{Gpa}$. The entire implant treated surface was within the block.

\section{Ball Abutments}

60 spherical abutments with the following characteristics were used: Diameter $2.25 \mathrm{~mm}$; Height $1.5 \mathrm{~mm}$. These abutments were hand torqued with the use a standard Astra Ball abutment driver. (Figure 3)

Random measurements of the ball abutment circumference were done in 10 randomly selected ball abutments with a Starrett Digital Micrometer, of accuracy $0.001 \mathrm{~mm}$. Measurements were done a priore and after testing. A Scanning Electron 
Microscope (four quadrant back scattered electron detector type 22, K.E. developments, LTD. Cambridge, England) was used to observe the surface of randomly selected new ball abutments and cycled ball abutments. (Figure 4 and Figure 5)

\section{Attachments}

30 Preci-Clix Astra female attachments were used. (Preat Corporation, Santa Ynez, CA). (Figure 6) This attachment is composed of two assembled components, a metal housing and a plastic insert. 30 additional inserts were used for a total of 60 inserts tested. The housing has retention elements for acrylic resin fixation. The insert has a snap-into function for optimal fit into the housing. (Figure 7)

\subsection{Attachment characteristics measured in a Photometric Analysis}

Prior to determining the angulations to be used between the implants and the attachments, a photometric analysis was done. This system had hardware capabilities such as a light microscope that was enabled to take pictures at desired magnifications. The resultant pictures could then be evaluated with the software for angulation, length, width, etc.

Pictures of the rotational limit of the Preci-Clix attachment over the spherical abutment where taken and then evaluated. First the attachment was placed onto the ball abutment parallel to the long axis of the dental implant and then at an angulated position to the point where the matrix contacted the stem of the abutment to which the 
sphere was connected. The resultant angle was $12.8^{\circ}$. It was assumed that the plastic insert could not flex to accommodate much more angle change. (Figure 8)

Furthermore the diameter of the plastic insert orifice was measured to evaluate how much it needed to distort to let the $2.25 \mathrm{~mm}$ sphere go through. (Figure 9)

\subsection{Attachment characteristics prior and after cyclic loading: a SEM evaluation}

Plastic inserts were evaluated microscopically prior to and after testing in order to visualize the morphologic changes that these components suffer during cycling.

Random selection of 5 new attachments was performed. Some factory discrepancies were noted. (Figure 10) After cyclic loading 1 attachment from every group was randomly selected and evaluated with a scanning electronic microscope. Wear in the plastic could be seen at different levels. (Figure 11)

\section{Lower Tool}

The G-10 Resin Block-Implant units were held in a professional work shop vise. This vise was screwed onto to a "Lexan" acrylic sheet platform. This base served as a clamping medium in order to attach the tool to the Instron Machine. The tool was acting as a mandible analog. (Figure 12) Implant angulation was controlled by means of prefabricated angled aluminum blocks, of $0^{\circ}, 10^{\circ}$ and $15^{\circ}$.(Figure 13 )

\section{Upper Tool}


A two piece aluminum device was fabricated with the intention to construct and test the attachment samples. This device served as a mandibular overdenture analog. It consisted of two pieces that were held together by means of bolts and washers. This split mold had two round receptacles in its lower area; they served as reservoirs where acrylic resin was flowed and the attachments were embedded. (Figure 14)

\section{Testing Machine}

Testing of the samples took place at the U.S. Army Dental and Trauma Research Detachment at Great Lakes, IL. An Instron Model 5581 (Instron Corp., Norwood, MA) with calibration date of $1 / 25 / 06$ was used. The capacity of the load cell used was $+/-50 N$. (Figure 15)

\section{Data acquisition software, testing protocol}

Licensed software property of the U.S. Army was used; Test Works 4®, Version 4.08 B Build 868 Copyright (C) 1995-2003 MTS Systems corporation. A systems engineer programmed the software according to the project's methods. Data was acquired at $10 \mathrm{hz}$ and the testing speed was $50 \mathrm{~mm} / \mathrm{min}$. Data was collected in terms of Peak Load and Valley Loads every 100 cycles, for a total of 36 data points

for each specimen. Peak Loads and Valley Loads were terms used for maximum pull load (tension) and maximum insertion loads (compression) respectively. Load was measured in Newtons and one cycle equaled one pull and one insertion. The software displayed the data in a graphical mode which was then retrieved manually and passed 
into spread-sheet forms. A total of 3,500 cycles were performed which simulated 3 years of use. (Figure 16)

\section{Specimen Preparation}

\subsection{Samples Description}

The experimental design included the construction of a simulated two implant retained overdenture model. 5 different angulation permutations between the abutments and the attachments were considered. These 5 configurations tested were: 1) $0^{\circ}$ implants- $0^{\circ}$ attachments $\left.(\mathrm{N}=6) ; \underline{2}\right) 10^{\circ}$ implants- $0^{\circ}$ attachments $\left.(\mathrm{N}=6) ; \underline{3}\right) 15^{\circ}$ implants- $0^{\circ}$ attachments $(\mathrm{N}=6) ;$ 4) $10^{\circ}$ implants- $10^{\circ}$ attachments $(\mathrm{N}=6) ; \underline{5}_{1}$ 15 $^{\circ}$ implants- $15^{\circ}$ attachments $(\mathrm{N}=6)$. (Figure 17)

\subsection{Incorporating the attachment on the ball abutment}

All Preci-Clix attachments were attached to the ball abutments at $0^{\circ}$ from the vertical reference plane using a Parelleling Mandrel (Preat Corporation, Santa Ynez, CA). The G-10 Resin blocks base was held parallel to the horizontal and the attachment was brought down with a Dental Surveyor (Ney), and held in place to avoid any rotation with injected Vinyl Polysiloxane impression material (type II medium viscosity, Reprosil, Dentsply Caulk. Milford, DE). (Figure 18)

\subsection{Incorporating the angles to the attachments}


Once the attachments were set in a fixed position following the vertical axis with the use of a surveyor and paralleling mandrel, both G-10 blocks were transferred to a professional vise. At this point three different aluminum blocks with incorporated angles could be used; the 0,10 or 15 degrees. The slope of these will run parallel to the flat G-10 block base and in turn will give the angulation to the abutment and attachment which run perpendicular to the surface of the aluminum block. (Figure 19)

Once the implants and attachments complex had been positioned on the preestablished inter-abutment distance of $20 \mathrm{~mm}$ on a predetermined angled block, the vise was closed and the attachments were ready to process following the implant angulation.

\subsection{Attachment processing}

Once the vise that was clamping the implant-attachment complex had been set satisfactorily at the pre-established inter-abutment distance and angle; the Upper split aluminum tool was lowered by means an Instron Grip which in turn was being held by a drill chuck of a professional Craftsman drill press. (Figure 20)

The vertical level as to which the tool needed to be lowered was locked on the drill press. No contact between the aluminum receptacles and the attachments was assured. Duralay clear acrylic resin (Reliance dental Mfg Co, Worth. ILL) was flowed into the receptacles and the top half of the tool was screwed together with bolts and washers. The overdenture analog was held into position until the acrylic was set. (Figure 21) 
The vise was then opened and the grip rose. As a result the attachments had the same angulation as the implants. This same process was followed when fabricating samples for all groups. 0,10 and 15 angled blocks were used for this effect. After processing the attachments, they were labeled right and left and also placed in a box labeled with the angle to which it was processed.

30 attachments were processed by the same operator following the same protocol. 9 pairs of attachments were processed using the 0 degree block, 3 using the 10 degree and 3 pairs using the 15 degree block.

\section{Specimen Assembly for testing}

The $1^{\text {st }}, 4^{\text {th }}$ and $5^{\text {th }}$ groups were set up differently from the $2^{\text {nd }}$ and $3^{\text {rd }}$. For the later groups the angle variation of the implants to the attachments was modified by; first setting the implants at the desired angulation 10 or 15 degrees by using the angled aluminum blocks on the vise; secondly by selecting the respective processed 0 degree attachments and placing them on the split half overdenture analog, closing the tool and then attaching it to the grip of the Instron Machine.

The upper tool carrying the attachments was then inserted into the abutments and then cyclic loading took place. The result of this protocol was a modification on the angulation between abutments and attachments. (Figure 22)

Groups \#1, \#4 and \#5 used attachments processed following the same implant angulation; hence there was no angle variation between the parts. In other words in this samples the angle incorporated to the attachments when processed was going to be followed by the implant angulation.(Figure 23) 
The lower tool that was bolted to the Lexan sheet was clamped to the Instron Machine during cycling. (Figure 24).

\subsection{Changing the cycled plastic inserts for new ones}

For simplicity, instead of using 60 Preci-Clix attachments, a decision was made to use 30 Factory assembled housing units and process them to the required angulations as described previously in section 7.4. After the factory installed plastic inserts $(F)$ were evaluated, they were removed from the housing using a \#11 blade. 30 new plastic inserts (Changed Inserts or $(C)$ inserts) were used to replace the used ones. As a result of this change the attachments were divided into Factory and Changed subgroups which added together gave the total number of samples tested; 60 attachments.

\section{Statistical Analysis}

Data in terms of peak load and valley loads were acquired every 100 cycles per specimen. 36 data points per specimen were recorded. A total of 2,160 total points were gathered and tabulated in 60 Excel (Microsoft@ Excel 2002) spread sheets (one per specimen). (Table 1)

A factorial ANOVA analysis was performed (using SAS Version 8.2) on the primary outcome variable: peak load $(\mathrm{N})$, since more than two groups were being compared. The main effects being tested for were the angles at which the attachments and implant-abutment complex were fixed; additionally, the effect of the

cycle was considered. All post-hoc analyses used Scheffe's test. In order for this 
particular analysis to be valid, a certain set of assumptions must be met: 1) That the variances between groups being compared are equal and 2) the variable being measured is normally distributed.

Prior final statistical evaluation, a separate analysis of variances was performed on the Factory tested plastic inserts $(F)$ and the Changed tested plastic inserts $(C)$; with the objective of establishing similarity between the two covariates before pooling them together to perform the final statistical analysis. 


\section{RESULTS}

Prior statistical evaluation of the available data; the assumption that the Changed $(C)$ inserts were the same as those from the Factory $(F)$ installed was evaluated. Looking at the overall means for peak load for the factory-installed and changed attachments (Table A) reveals that the mean for the factory-installed attachment was lower, and the variance was larger.

\begin{tabular}{|l|r|r|r|r|}
\hline Attachment Type & Mean & Median & Std Dev & Variance \\
\hline Changed Attachment & 20.8833519 & 21.0430000 & 2.2882833 & 5.2362406 \\
\hline Factory Attachment & 17.9374630 & 17.9345000 & 2.8977612 & 8.3970201 \\
\hline
\end{tabular}

Table A. Summary Statistics for Peak Load by Condition

If we compare the means for each condition by attachment (Figure A) we see that the mean peak load for the factory attachment was lower than when the attachment was changed. Except for the factory attachment for the 15-0 condition, the overall trend appears the same.

Mean PEAK_LOAD vs. CONDITION

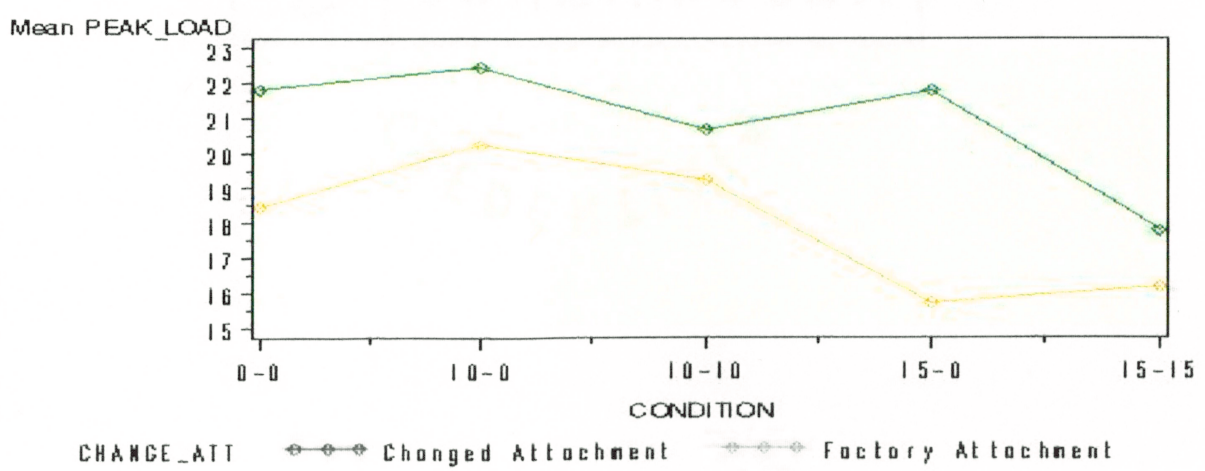

Figure A. Means of the various conditions and attachment types. 
As seen in table $\mathrm{B}$, the variances for the factory attachments tend to be larger, although not by much, for all the conditions with the exception of the 10-10 degree sample which has low variance. The 15-0 factory attachment denotes the highest variance of all, and in addition shows mean retention discrepancies when compared with the changed attachments.

\begin{tabular}{|c|l|r|r|r|r|}
\hline CONDITION & $\begin{array}{c}\text { VARIABLE: } \\
\text { CHANGED/FACTO } \\
\text { RY }\end{array}$ & $\begin{array}{c}\text { Mean } \\
\text { PEAK_LOAD }\end{array}$ & $\begin{array}{c}\text { Median } \\
\text { PEAK_LOAD }\end{array}$ & $\begin{array}{c}\text { STD } \\
\text { PEAK_LOAD }\end{array}$ & $\begin{array}{c}\text { Var iance } \\
\text { PEAK_LOAD }\end{array}$ \\
\hline $\mathbf{0 - 0}$ & Changed Attachment & 21.79 & 21.38 & 1.36 & 1.87 \\
\hline & Factory Attachment & 18.44 & 17.68 & $\mathbf{2 . 6 9}$ & $\mathbf{7 . 2 6}$ \\
\hline $\mathbf{1 0 - 0}$ & Changed Attachment & 22.42 & 22.89 & 1.57 & 2.48 \\
\hline & Factory Attachment & 20.20 & 19.88 & $\mathbf{1 . 7 1}$ & $\mathbf{2 . 9 4}$ \\
\hline $\mathbf{1 5 - 0}$ & Changed Attachment & 21.77 & 21.17 & 1.47 & 2.17 \\
\hline & Factory Attachment & 15.68 & 17.31 & $\mathbf{3 . 2 1}$ & $\mathbf{1 0 . 3 1}$ \\
\hline $\mathbf{1 0 - 1 0}$ & Changed Attachment & 20.66 & 20.39 & 1.49 & 2.24 \\
\hline & Factory Attachment & 19.20 & 18.61 & 1.34 & 1.80 \\
\hline $\mathbf{1 5 - 1 5}$ & Changed Attachment & 17.76 & 16.03 & $\mathbf{2 . 1 3}$ & 3.68 \\
\hline & Factory Attachment & 16.14 & $\mathbf{4 . 5 4}$ \\
\hline
\end{tabular}

Table B. Various Summary Statistics for Peak Load by Condition and Attachment Type

Figure $\mathrm{B}$ shows that as the mean increases, the sample standard deviation decreases: in other words, for this data, the mean and variance are inversely proportional. It is not clear, if this relationship is statistically significant, and poses a threat to the assumption; although the large variances observed for the 0-0 degree and 15-0 degree groups do violate this assumption. 


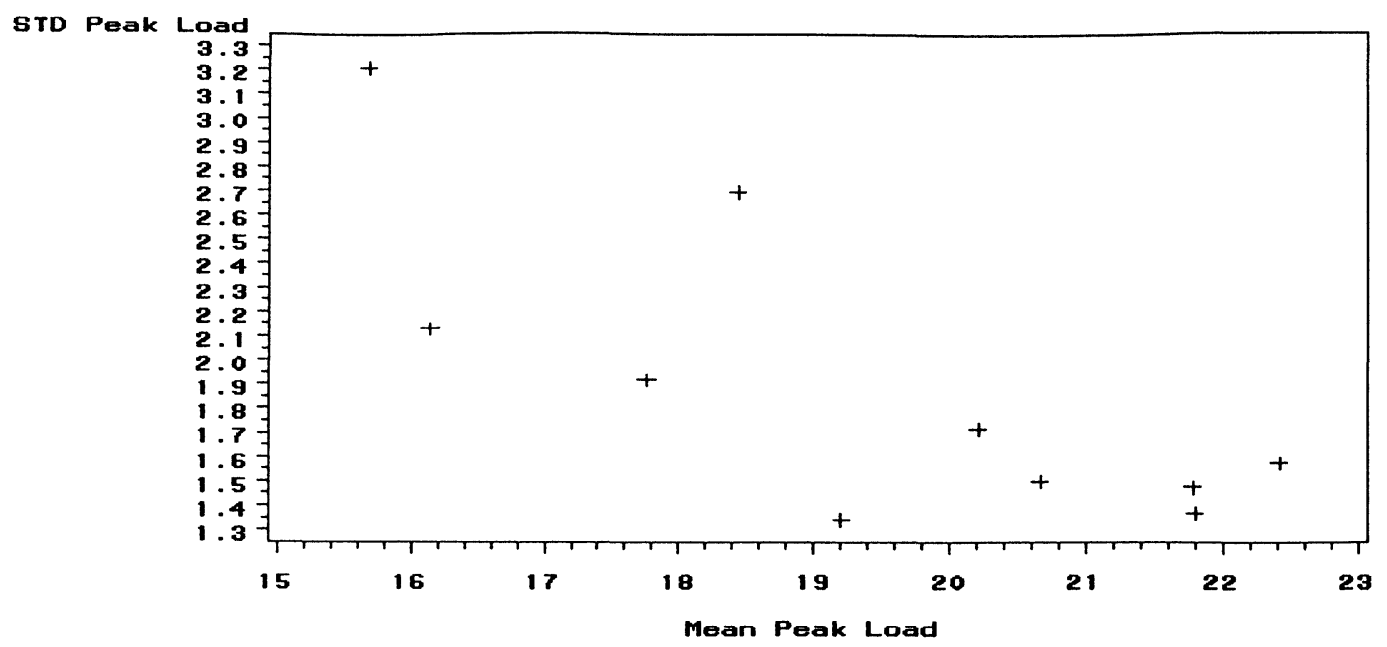

Figure B. Plot of the Standard Deviation vs. Mean for Attachment types and Conditions.

\section{Normality of Data}

Figure $\mathrm{C}$ below shows the observed distribution of peak load in the data. The data is skewed with a large percentage of low values. A variety of transformations were considered (e.g. log transformation), but none proved satisfactory either in their results or in the ease of interpretation.

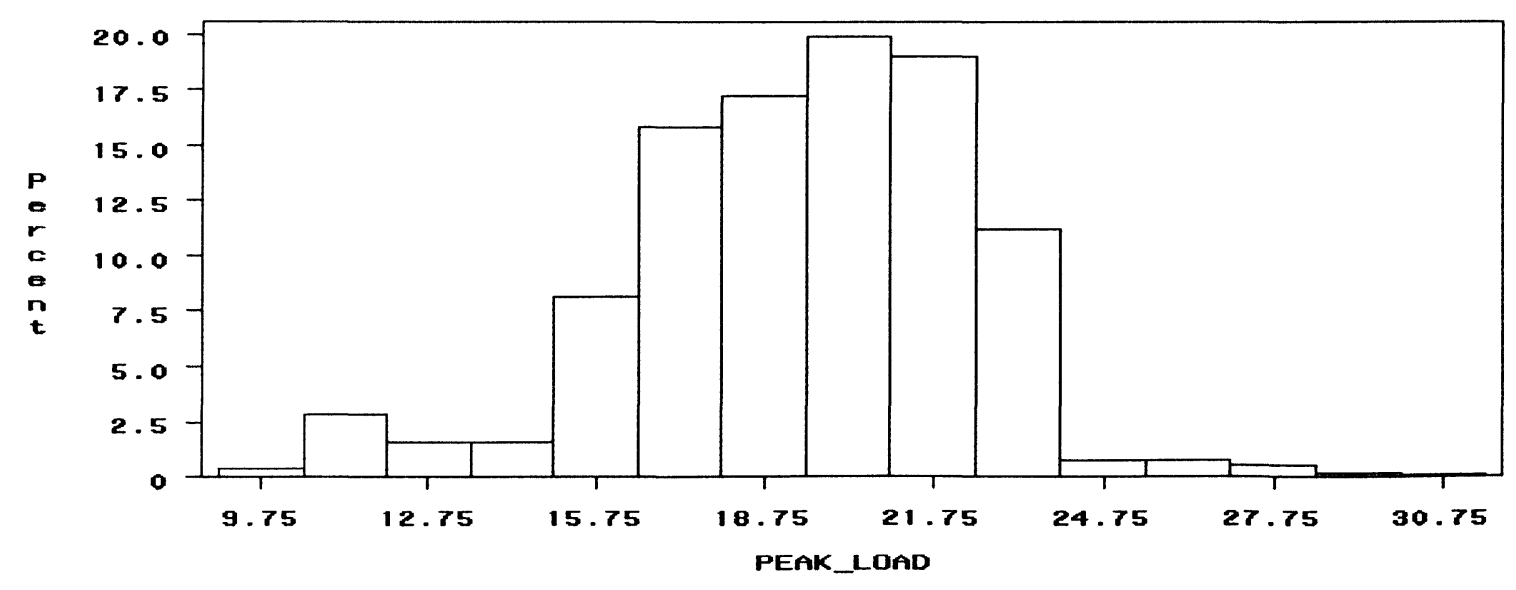

Figure C. The distribution of Peak Load is skewed. 


\section{Variance Structure of Data}

When considering the experimental conditions the variance of $15-0$ seems to be high compared to the other ones. This could be attributed to the Factory installed attachment covariate. See table C.

\begin{tabular}{|l|r|r|r|r|}
\hline $\begin{array}{l}\text { Experimental } \\
\text { Condition }\end{array}$ & Mean & Median & Std Dev & Variance \\
\hline $0-0$ & 20.1177176 & 21.1310000 & 2.7148650 & 7.3704917 \\
\hline $10-0$ & 21.3144861 & 21.2070000 & 1.9827692 & 3.9313739 \\
\hline $15-0$ & 18.7341528 & 20.1550000 & $\mathbf{3 . 9 4 0 9 6 5 5}$ & $\mathbf{1 5 . 5 3 1 2 0 8 8}$ \\
\hline $10-10$ & 19.9323981 & 19.9695000 & 1.5961892 & 2.5478200 \\
\hline $15-15$ & 16.9532824 & 17.2385000 & 2.1798918 & 4.7519281 \\
\hline
\end{tabular}

Table C. Summary Statistics for Peak Load by Condition

While ANOVA analysis is robust against departures of normality and variance, the variance structure in this data appears, for some conditions, to be out of limits. An alternative analysis would have been a non-parametric analysis, but these depend on equality of variances between groups; and this is clearly not the case here. Bearing these caveats in mind and for ease of interpretation, the ANOVA analysis was carried out. The results are given below.

\section{Results of ANOVA analysis:}


Below is given the overall ANOVA table for the analysis. We have statistically significant evidence that there is a difference in at least one of the conditions being tested $(\mathrm{p}<0.0001)$.

\begin{tabular}{|l|r|r|r|r|r|}
\hline Source & DF & Sum of Squares & Mean Square & F Value & Pr > F \\
\hline Model & 40 & 5751.771281 & 143.794282 & 37.92 & $<.0001$ \\
\hline Error & 1039 & 3939.686794 & 3.791806 & & \\
\hline Corrected Total & 1079 & 9691.458075 & & & \\
\hline
\end{tabular}

Table D. Overall ANOVA table

The tables below give the results for tests of the main effects: condition, changed attachment, and cycle. There is a difference in means in the cycle, although the current analysis is not designed to determine trends. There is also statistically significant evidence that at least on of the conditions is different from the other.

\begin{tabular}{|l|r|r|r|r|r|}
\hline Source & DF & Type I SS & Mean Square & F Value & Pr > F \\
\hline CONDITION & 4 & 2352.901248 & 588.225312 & 155.13 & $<.0001$ \\
\hline CHANGE_ATT & 1 & 2343.130563 & 2343.130563 & 617.95 & $<.0001$ \\
\hline CYCLE & 35 & 1055.739470 & 30.163985 & 7.96 & $<.0001$ \\
\hline
\end{tabular}

Table E. ANOVA results for main effects

Table $\mathrm{F}$ below gives the results of Scheffe's post-hoc test. There are differences between all the conditions (indicated by $\mathrm{X}$ ) except between the 0-0 degree and the 10-10 degree. This is somewhat unexpected, however, looking at table A and figure $\mathrm{D}$ below, is not surprising given the data. 


\begin{tabular}{|c|c|c|c|c|c|}
\hline & $0-0$ & $10-0$ & $15-0$ & $10-10$ & $15-15$ \\
\hline $0-0$ & & & & & \\
\hline $10-0$ & $\mathrm{X}$ & & & & \\
\hline $15-0$ & $\mathrm{X}$ & $\mathrm{X}$ & & & \\
\hline $10-10$ & & $\mathrm{X}$ & $\mathrm{X}$ & \\
\hline $15-15$ & $\mathrm{X}$ & $\mathrm{X}$ & $\mathrm{X}$ & $\mathrm{X}$ & \\
\hline
\end{tabular}

Table F. Results of Scheffe's Post-Hoc Tests

\section{Mean PEAK LOAD vs. CYCLE}

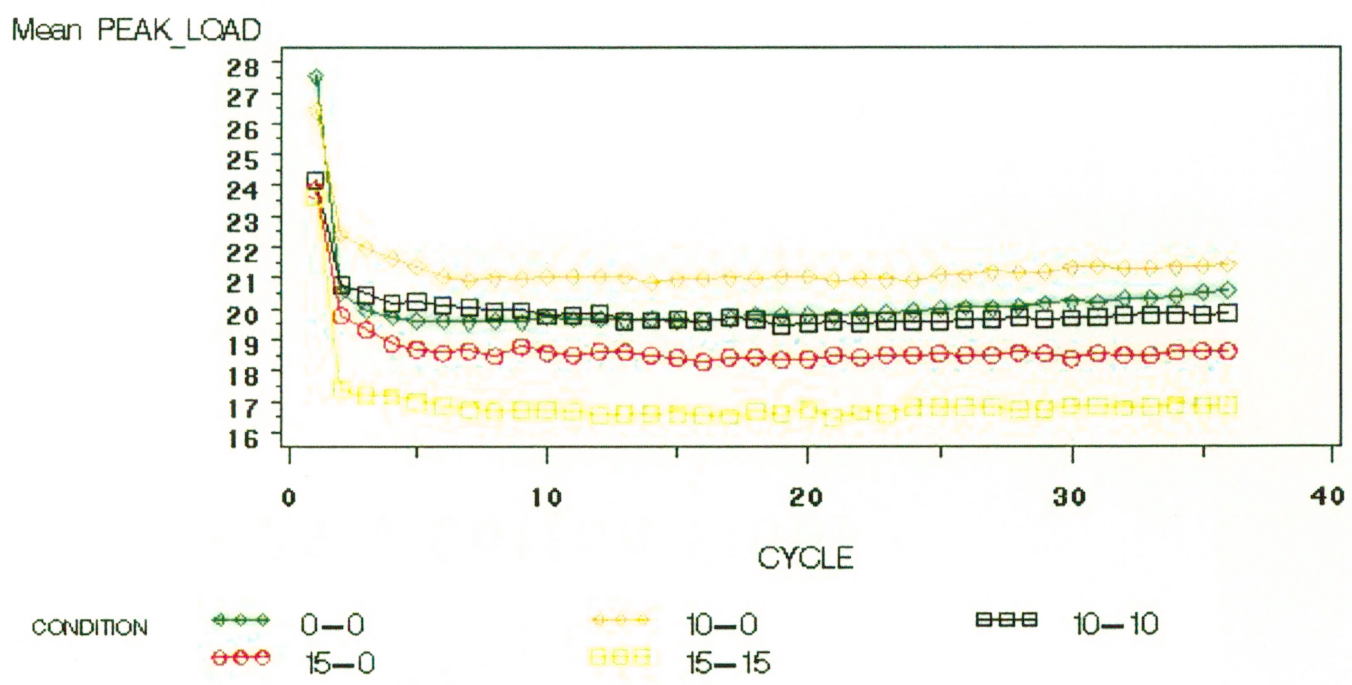

Figure D. Graph of mean peak load for each condition over the 3500 cycles.

With this result in mind the following contrasts were also tested in the context of the ANOVA model. There is significant statistical evidence that Condition 3 is different from Conditions $1 \& 2(\mathrm{p}<0.0001)$; and that Conditions 4 $\& 5$ are different from Conditions $1 \& 2(\mathrm{p}<0.0001)$. See table $\mathrm{G}$. 


\begin{tabular}{|l|r|r|r|r|r|}
\hline Contrast & DF & Contrast SS & Mean Square & F Value & Pr > F \\
\hline Condition 1 \& 2 vs. 3 & 1 & 88.443575 & 88.443575 & 23.32 & $<.0001$ \\
\hline Condition 1 \& 2 vs. 4 \& 5 & 1 & 1782.127728 & 1782.127728 & 469.99 & $<.0001$ \\
\hline
\end{tabular}

Table G. Differences between conditions 


\section{DISCUSSION}

The success of implants to improve functional deficiencies of traditional removable prosthesis therapy for edentulous patients has made their use popular. Although several authors have complained of maintenance issues with ballattachment systems and of difficulty of use in a non-parallel implant scenario[20, 62, 64]; they have become the most preferred retentive structure in overdentures.[56, 63, $65,66,77]$

Current advances in dental technology, have introduced to the overdenture modality the use of two piece retentive systems, for instance; Locator, ERA and Preci-clix attachments. Some authors have empirically stated that these systems are an improvement in maintenance protocols when compared to standard gold matrices. The trend is to use attachment systems consisting of two parts; a housing that is embedded on the prosthesis intaglio surface and a replaceable plastic retentive insert. $[56,63,81]$ The insert can be replaced with ease when compared to a one piece attachment system like the gold matrix.

The present in vitro study investigates the retention properties of Preci-Clix spherical attachments on different angulation permutations between them and the implants. The primary objective of this study is to evaluate whether divergent implants could be predictably managed in terms of retention with parallel attachments when compared to a parallel implant scenario. A related objective was to evaluate the attachments behavior in terms of retention when processed following a divergent implant overdenture situation. 
Authors have done research on the retentive behavior of ball attachments on parallel implants; $[69,75,77]$ only one article, in a one implant overdenture model, has described the effect of implant angulation and retentiveness on parallel attachments.[65] To the author's knowledge, to date there is no research that merges retentiveness characteristics and angle variability between parallel and non-parallel implants and parallel and non parallel attachments.

This project also evaluates, the clinical observations made upon the rotational capability of spherical attachments when used to compensate non-parallel implants.[66]

Some authors and stud attachment manufacturers have advocated the use of this retentive modality in clinical situations where the implants are placed parallel to one another exclusively, in order to prevent premature wear of the involved components and to obtain predictable attachment retention.[61, 82, 83] Moreover, authors that believed in this theory used alternative attachment systems such as magnets when faced with non-parallel implants.[84] Likewise, Zarb et al, concluded in their longitudinal study that; non-parallel implants should be treated with bar and clip prostheses. Although this study observed decreased retention in gold matrices when these were used in a non-parallel implant scenario; he ignored the attachment position relative to the implant. [85]

Bar and clip retained and supported overdentures require more restorative space, are initially more expensive and technically more complex than free standing attachment systems.[56] One of the aims of this study was to illustrate the potential 
for use of the rotational capability concept of spherical attachments to correct nonparallel implant angulations.

The present study analyzed the attachment retentive behavior in instances where the implants were parallel or non-parallel to one another. The degrees of divergence are described relative to the vertical reference plane. In samples 1,2 and 3 the attachment was left parallel to the vertical reference plane and the implant angulation was varied to 0,10 and 15 degrees respectively from the vertical reference plane. The mean peak load for the $1^{\text {st }}$ group samples $\left(0^{\circ}\right.$ implant $-0^{\circ}$ attachment $)$ was $20.11 \mathrm{~N}$, meanwhile for the $2^{\text {nd }}$ group (10 implant $-0^{\circ}$ attachment) was $21.31 \mathrm{~N}$. Although the loads seem to be close to one another other, statistically there is a difference between groups when looking at the Scheffe's post-hoc results.

The mean retention value of the $3^{\text {rd }}$ group $\left(15^{\circ}\right.$ implants- $0^{\circ}$ attachments $)$ was 18.73 N, which when compared to the $1^{\text {st }}$ group's mean; $20.11 \mathrm{~N}$, yield statistical significant differences $(\mathrm{P}<0.0001)$. Despite the application of the rotational capability concept in the correction of a non-parallel implant situation in groups \#2 and \#3; the retentive values seem to decrease slightly with the implant angle modification when compared to group \#1. Although minimal changes are observed between the $3^{\text {rd }}$ and $1^{\text {st }}$ group one can hypothesize that the reduction in the $3^{\text {rd }}$ group is due to the fact that the Preci-clix attachment is being tested beyond its rotational threshold which might prevent complete seating of the plastic insert above the ball's height of contour. This maximum ability to rotate was evaluated in a photometric analysis and it was found to be approximately $12.8^{\circ}$. (measured in a one abutment-attachment configuration) [86] Clinical applicability of these results suggest that the Preci-Clix spherical attachments 
retention is sacrificed when used in situations where the implants are divergent to each other beyond $25.6^{\circ}$ (total)

Variability between the means was observed on the $1^{\text {st }}$ group. A reason for this phenomenon could be that the factory $(F)$ incorporated plastic inserts showed variability when compared to the changed $(\mathrm{C})$ acrylic inserts. Variance in the factory assembled attachments was seen consistently in all groups. It seemed that changing the used attachments for new ones, reduced variance and increased retention. In hindsight it would have been ideal to standardize all attachments into one of the two variables; factory assembled or manually changed. Nevertheless authors have applied this protocol in clinical prospective studies as well. [81]

The mean retentiveness for the factory installed plastic inserts for all samples was lower when compared to the changed plastic inserts; $17.93 \mathrm{~N}$ and 20.88 respectively. These observations applied to a clinical scenario where the plastic inserts are changed for new ones once the factory installed ones loose retention, suggests that the changed inserts could be more retentive, which in turn will convey more stability and possibly more patient satisfaction.

Botega and coworkers found after cyclic loading; similar retentive values as those found in this present study. Their results on ball abutments ranged from $18.4 \mathrm{~N}$ to $21.09 \mathrm{~N}$. Nevertheless parallel implants and parallel attachments were used in that study.[80]

In the current study, the first pull loads observed in all specimens are considerably higher than subsequent measurements. This "one pull" observations suggests that the assumptions made with using single pull studies are not what might 
be expected on a clinical scenario, where the overdentures are removed constantly. This methodology $[76,77,87,88]$ can not provide evidence about maintenance nor patient satisfaction or reliable consistency of retention.

The present in-vitro study incorporated cyclic loading of the attachments at different angulations. Only a few authors used cyclic loading as the protocol for their studies. $[73,75,80]$ The rationale behind cyclic loading was that the attachment behavior throughout time varies as the components wear, deform or undergo intrinsic physical changes.

In a more recent in vitro study by Rutkunas and Mizutani;[87] stud and magnet attachments where evaluated with a mandibular overdenture analog that was pulled once in different directions in an effort to assess retention and stability. The authors in their discussion recognized the need for studies to clarify the range of retention and fatigue behavior of overdenture attachments.

Clinical studies have tried to incorporate single pull tests at baseline and after months of use. Van Kampen, et al [78] evaluated in vivo the retention and maintenance of three mandibular overdenture attachment systems. They had 6 patients per group; retention was evaluated by a hybrid of strain gauges at base line and after 3 months of use. Retention values were found to be $29.7 \mathrm{~N}$ for stud attachments. However sample size and number of data points collected per sample, reduce the power of this study.

The present study follows and expands an original concept explained by Weimeyer's and co-workers.[66] Their proposal of implant angulation correction with the use of parallel attachments was carried out using Straumann Ball abutments 
and Gold matrices. They outlined that the rotational capability of these attachments could potentially correct up to $30^{\circ}$ off-axis angulation of the vertical reference plane per implant. Their observations were made on the assumption that when the matrix is rotated on a ball abutment, the moment it contacts the stem that holds the abutment's sphere, was considered as the rotational limit and if measured in degrees, it will yield maximum angulation correction capability. Although a conceptually valid observation, additional research was advocated.[66]

As mentioned previously the current study used a photometric analysis to evaluate the Preci-Clix attachment rotational capability. As opposed to the $30^{\circ}$ rotational capability of the gold matrix (per implant) mentioned in Weimeyer's work; the Preci-Clix spherical attachment was found to have approximately $12.8^{\circ}$ of correction potential per implant.

Another recent in vitro study, Gulizio and co-workers[65] in a one implant overdenture model,[65] evaluated 4 Gold and 4 Titanium matrices placed at $0^{\circ}$ in respect to 4 ball abutments positioned at $0^{\circ}, 10^{\circ}, 20^{\circ}$ and $30^{\circ}$ from the vertical reference plane. Results from a one pull test and factorial peak retention data yield higher retentive values for gold matrices tested in a $0^{\circ}$ abutment scenarios when compared to the ones evaluated in $30^{\circ}$ abutment configurations. The mean retention values were $26.7 \mathrm{~N}$ and $21.4 \mathrm{~N}$ respectively. Their conclusions were that the angulation factor has an effect on retentiveness of gold matrices. Titanium matrices results followed the same trend, but with even lower values on the $30^{\circ}$ abutment; they had mean loads of $18.3 \mathrm{~N}$. 
Even though their results yield statistical differences in terms of retention when taking angulation as a factor; categorical assumptions could not be accounted for; due to its design limitations; for instance the small sample size of 1 specimen per condition that resulted in high variances when subject to a factorial analysis. On the other hand, the authors stated that their model could be used in cyclic testing which would be relative to a clinical scenario. Nevertheless the current study follows their lead for future research that Gulizio and co-workers outlined at the end of their work.[88]

Results of the present study observed in the $1^{\text {st }}, 2^{\text {nd }}$ and $3^{\text {rd }}$ groups, follow similar trends observed in Guilizio[65] study. In the current study, the main effects such as condition (implant and attachment angulation), plastic insert characteristics (changed or factory) and cycle\#, were found to be statistically significant $(\mathrm{P}<0.0001)$

Recognition of retentiveness of mandibular implant retained overdentures as a predictor of patient satisfaction has driven several authors to test in-vitro retentiveness of different attachment systems.[39, 65, 74-76] All authors, except Gulizio and co-workers, did not account for frequently encountered clinical scenarios where mandibular implants placed for overdentures often end up being non-parallel to one another.

Common processing errors such as accidental attachment movement during acrylic resin trial packing and excessive acrylic polymerization shrinkage could potentially affect attachment retentiveness and seating accuracy of the patrix-matrix complex. Anusavice [89]stated that acrylic resin linear volumetric shrinkage is equivalent to $1 \%$ or less. This distortion could potentially affect the fit of metal to 
metal attachment systems like the ones described in Weimeyer's [66] and Gulizio's [65] articles. The current study uses a polymer based Preci-Clix attachment system which allows for some degree of plastic deformation and recovery.

The current study also evaluated a very common clinical scenario where implants are placed in certain degree of divergence and their respective attachments are processed following their long axis angulation. Observations made in a preliminary study by Wiemeyer, Agar and coworkers,[66] outlined the mechanical issues related to attachments processed in the previously described manner; they stated that each matrix would not engage sufficient undercut medially but would engage excessive undercut laterally, which would cause problems such as incomplete seating and premature wear.

This present study simulates these situations with groups 4 and 5 . These groups have implants placed at 10 and 15 degrees off the vertical axis, and attachments processed following their long axis angulation. (Figure 25) After cyclic loading, results show for the group 4, that there were not statistically significant differences between this group and group 1. When comparing their means $19.9 \mathrm{~N}$ and $20.1 \mathrm{~N}$ respectively, they show a narrow margin of difference. One can say that the Preci-Clix plastic attachments, when processed following a subtle non-parallel implant long axis, allows for acceptable functionality in terms of retention when compared to a parallel implant-parallel attachment scenario. These findings somehow contradict Wiemeyer's [66]observations. A logical explanation to this difference could be that their observations were made on a different attachment; the gold matrix; and the current study used a plastic insert as its retentive component. 
Apparently the plastic insert in the Preci-Clix attachments have inherent capabilities of flexing and recovering without undergoing permanent deformation. This is true at least for the $4^{\text {th }}$ configuration which gives more capacity for the external walls of the attachment to accommodate in and out of the abutment without a significant change in retention.

The results for the $5^{\text {th }}$ group; the 15-15 implant-attachment configuration, showed the lowest retention of all groups, contrary to what was expected in our hypothesis which was made primarily on the basis of the gold matrices used in Wiemeyer's [66]and Gulizio's[65] studies. It was anticipated that the Preci-Clix matrices were going to be able to go in all the way and that the degree of divergence would have made its removal harder than the other groups studied. As explained previously this assumption was sustained by Wiemeyer's technique article. This group's peak mean values were $16.9 \mathrm{~N}$. Almost $4 \mathrm{~N}$ below the $1^{\text {st }}$ group mean. However, after 100 cycles the retentive values in this group stabilized at this lower level of retention and remain constant throughout the test.

Further evaluation of the $5^{\text {th }}$ group samples by electron microscopy after testing, revealed permanent plastic deformation on the outer aspect of the retentive component and structural damage of the of the plastic insert.(Figure 26) These observations were not consistent with the SEM images obtained from the other groups. Further SEM analysis of the medial aspect of the plastic inserts, contra lateral to the deformed area of this group reveals fewer changes. (Figure 27) This permanent plastic deformation observed is consistent with excessive contact and pressure with the external or outer side of the ball abutment. 
A similar pattern of observations was seen in the 15-0 configuration. Where the outer aspect of the attachment was not engaging much beyond the height of contour of the sphere and the internal aspect was being forced into the undercut and onto the stem that supported the ball.(Figure 28) SEM analysis of plastic inserts exemplifies more wear on the inner aspect, compared to the outer surface.(Figure 29) A greater magnification showed, loose pieces of plastic accumulating on the outer rim.(Figure 30) The mean peak values of this group were also lower than the $1^{\text {st }}$ group; $18.7 \mathrm{~N}$. The stabilization of retention under these conditions is difficult to explain

Besides considering cycling as a covariate and in an effort to replicate the use of attachments in the oral cavity, authors have also incorporated a wet environment to their testing; for instance Botega DM, et al,[80] in a study evaluating retention force and fatigue of ball and bar and clip attachments, tested their 4 samples, submerged in a saliva substitute. In a similar study Setz, et al [75] used saliva substitute at $22^{\circ} \mathrm{C}$.

Although the present study did not use saliva, its use might have been a step closer to a clinical situation although the Preci-clix plastic insert is said to be waterproof; the moisture might lubricate the contacting parts and affect loads.

Although statistically significant differences were found between the $1^{\text {st }}$ and $2^{\text {nd }}$ groups, their mean peak results; $20.1 \mathrm{~N}$ and $21.3 \mathrm{~N}$ suggest that non-parallel implants could be successfully managed be processing spherical Preci-clix attachments following the path of overdenture removal. Nevertheless the $3^{\text {rd }}$ configuration yield lower retentive peak values, which suggests given the stated evidence, that 15 degree implants are beyond the rotational capability of the Preci- 
Clix attachment hence they could potentially lose retention at a faster rate when compared to the $1^{\text {st }}$ and $2^{\text {nd }}$ groups. This could result in an increased frequency of maintenance in a clinical situation.

This study suggests that a 10 degree off axis implant situation or 20 degrees between two implants, could be handled predictably by using either attachments processed following the implants long axis, or by placing attachments parallel to each other following the path of removal of the prosthesis. Although the nature of forces acting on an overdenture is far more complex than this in vitro study, the results suggest that the plastic inserts are more forgiving that one might think.

The 15-15 configuration yielded the least retentive values. The stated evidence suggests that processing the matrices following this extreme off-axis angulation will destroy the attachments upon function. The results suggest that if there is more than 30 degrees of difference between implants placed for an overdenture, alternative attachment systems can be then used such as bars, clips or magnets.

With the aim of evaluating long term maintenance with the Preci-Clix attachments, fatigue testing was accomplished. Results revealed possible sources of an increased maintenance frequency. Groups 3 and 5 yielded the lowest mean peak values after 3,500 cycles; $18.7 \mathrm{~N}$ and $16.9 \mathrm{~N}$ respectively. The trend seems to be an initial reduction of their retentiveness but then the amount of retention stabilized. This would indicate satisfactory clinical performance. However, SEM evaluation denotes permanent plastic deformation and loss of structure in various samples randomly selected from these groups. 
For the $3^{\text {rd }}$ group, evidence suggests that spherical Preci-Clix attachments that are processed parallel to their vertical reference plane to implants that are beyond their rotational capabilities, $\left(12.8^{\circ}\right)$ have the potential to lose some initial retentiveness and may show signs of deterioration, although its overall retentiveness stabilized passed 100 cycles.

In the $5^{\text {th }}$ group, effects of extreme implant angulation with attachments processed following the abutments long axis, are not what was expected initially. They yield the lowest retention and showed a substantial amount of plastic deformation. Clinical implications of these findings are difficult to predict since this is a controlled environment and the human oral cavity is not. There is a multifactorial array of conditions that affect retention of attachment systems in vivo, vertical forces is one of those.

Prospective and retrospective reports of post-insertion complications with implant retained overdentures are numerous. The consensus of some studies is that maintenance requirements are the greatest during the first year of service.[90-92]

Gotfredsen and Holm[70] in a randomized prospective 5 year study concluded that frequency of technical complications/repairs per patient was higher around bars when compared to ball attachments. In another study, Walton and coworkers[69] recorded one year prosthetic outcomes of implant overdentures. She found that there were a significant number of repairs for the Titanium attachments. These attachments are composed of three pieces; a housing that remains embedded in the overdenture intaglio surface and a threaded second housing that holds a $\mathrm{C}$ steel spring. Walton found that the spring was breaking frequently, and needed to be replaced often and 
that the ball abutments where getting lose. This specific spherical system proved in this, and other studies by the same author,[67] to be less efficient when compared to bars and clip systems. One major factor that could have influenced the high frequency of abutment loosening, was that external hexed implants were used in her study.

In terms of maintenance the Titanium attachments have performed unreliably in clinical evaluations performed by Walton, nevertheless ball attachment systems continue to be used with more frequency than bar systems.

Only a few studies have related implant angulation variability and attachment angulation variability, to retentive forces and maintenance issues. $[65,66]$ The results of the present study suggest that a higher than normal maintenance frequency could be a result of erroneous attachment position with respect of implant angulation in mandibular overdentures. Some evidence was found that attachments are permanently damaged when they are processed and cycled in extremely divergent implant scenarios ( $5^{\text {th }}$ group). However, the finding that retentive values stabilized suggests that the clinical performance could be satisfactory for these attachments under the conditions tested.

This in vitro research project applies to most $2.25 \mathrm{~mm}$ spherical abutments, to mention some: Astra, Straumann and Nobel Biocare (Replace Select).

Although this study validates the rotational corrective capability of spherical attachments and warns about their use in extreme implant divergence; the dynamic nature of the mandibular overdenture involves many more conditions than the ones evaluated in this investigation. Function varies with individual residual ridge 
anatomy, muscle attachment levels. Occlusion, diet and tissue resiliency are some of the confounding factors that have the potential to affect overdenture retention and stability. 


\section{CONCLUSIONS}

Relative to the conditions of this study the following conclusions where made:

1. The retention of Preci-Clix attachments processed parallel to the vertical reference plane is not affected when used in implants with a $20^{\circ}$ divergence configuration. Hence attachments could be rotated on the spherical abutment to the path of insertion and removal of an overdenture to compensate for moderate implant non-parallelism without significantly sacrificing retention.

2. Preci-Clix attachments when used parallel to each other in an aim to correct a $15^{\circ}$ implant divergence yield lower retentive values to those of the parallel implantparallel attachment configurations.

3. Preci-Clix matrices processed following the long axis of $10^{\circ}$ divergent implants can be predictable in terms of retention.

4. Preci-Clix attachments processed following the long axis of $15^{\circ}$ divergent implants are not predictable in terms of retention and have the potential to have maintenance problems.

5. The retention for all attachments tested stabilized after initial loss of retention. 


\section{FUTURE RESEARCH}

The experimental design used in this research project could be used for future testing. It is unique because samples could be built and tested in the same model.

Future testing could include:

1. A wet environmental chamber could be used to test the samples. This would more closely simulate the oral environment.

2. The plastic recovery, permanent deformation and wear characteristics of the plastic inserts could be compared to a more rigid attachment system, like the gold matrix in bar in non-parallel and parallel implant scenarios.

3. During cyclic loading, a longer period of time could be left between cycles to evaluate if the plastic insert might recover differently.

4. The same setup could be used to evaluate a variety of attachment systems and angles in a fatigue testing mode. 


\section{IX.BIBLIOGRAPHY}

1. CDC, Public Health and Aging: Retention of Natural Teeth Among Older Adults---United States, 2002, in MMWR weekly. 2003. p. 1226-1229.

2. Burt BA and Eklund SA, Dentistry, dental practice, and the community. 5th Edition ed. 1999, Philadelphia, Pennsylvania: WB Saunders Co.

3. Marcus SE, et al., Tooth retention and tooth loss in the permanent dentition of adults: United States 1988-1991. J Dent Res, 1996. 75 (Spec Iss): p. 684-695.

4. CDC, Surveillance for Dental Caries, Dental Sealants, Tooth Retention, Edentulism, and Enamel Fluorosis in the United States, 1988-1994 and 19992002, in Surveillance Summaries. 2005, CDC. p. 1-44.

5. Douglass CW, Gammon MD, and Atwood DA, Need and effective demand for prosthodontic treatment. J Prosthet Dent, 1988(59): p. 94-104.

6. Douglass $\mathrm{CW}$ and Watson AJ, Future needs for fixed and removable partial dentures in the United States. J Prosthet Dent, 2002(87): p. 9-14.

7. Douglass $\mathrm{CW}$, Shih A, and Ostry L, Will be there a need for complete dentures in the United States in 2020? J Prosthet Dent, 2002. 87(1): p. 5-8.

8. Marcus PA, et al., Complete edentulism and denture use for the elders in New England. J Prosthet Dent, 1996: p. 260-266.

9. Gift HC and Redford M, Oral Health and the quality of life. Clin Geriatr Med, 1992(8): p. 673-83.

10. Hinds K and Gregory JR, National diet and nutrition survey: People aged 65 years or over: Vol 2; Report of the oral health survey. London: Stationary Office. 1998. 
11. Feine JS and Carlsson GE, Implant Overdentures: The Standard of Care for Edentulous Patients. 1st ed. Edentulism, Digestion, and Nutrition, ed. T.J. Morais JA. 2003, Hong Kong: Quintessence Books.

12. Zarb GA, et al., Prosthodontic Treatment for Edentulous Patients. 12th ed, ed. Zarb GA and Bolender CL. 2004, St. Louis, MO: Mosby.

13. Van Wass MAJ, The influence of clinical variables on patient satisfaction with complete dentures. J Prosthet Dent, 1990. 63: p. 307-310.

14. Batenburg RHK, et al., Treatment Concept for Mandibular Overdentures Supported by Endosseous Implants: A literature review. Int J Oral Maxillofac Implants, 1998. 13: p. 539-545.

15. Tallgren A, The continuing reduction of the residual alveolar ridges in complete denture wearers: A mixed longitudinal study covering 25 years. J Prosthet Dent, 1972. 27(2): p. 120-132.

16. Fenton AH, The decade of overdentures: 1970-1980. J Prosthet Dent, 1998. 79: p. 31-36.

17. Atwood DA, Reduction of residual ridges: a major oral disease entity. $\mathrm{J}$ Prosthet Dent, 1971. 26: p. 266-79.

18. Carlsson GE, Clinical morbodity and sequelae of treatment with complete dentures. J Prosthet Dent, 1998. 79: p. 17-23.

19. Zarb GA, Introduction to the Halifax Symposium "Towards Optimized Management of Edentulous Predicament". J Prosthet Dent, 1998. 79: p. 3-4.

20. Mericske-Stern $\mathrm{RD}$, Overdentures with roots or implants for elderly patients: A comparison. J Prosthet Dent 1994;72:543-50, 1994. 72: p. 543-550. 
21. Preiskel HW, Overdentures made easy. 1996, Berlin: Quintessence books.

22. Brill N, Adaptation and the hybrid prosthesis. J Prosthet Dent, 1955. 5: p. $811-23$

23. Miller, P.A., Complete dentures supported by natural teeth. J Prosthet Dent, 1958. 8: p. 924-8.

24. Prince JB, Conservation of the supportive mechanism. J Prosthet Dent, 1965. 19: p. 327-38.

25. Crum RJ and Rooney GE, Alveolar bone loss in overdentures: a 5 year study. J Prosthet Dent, 1978. 40(6): p. 610-613.

26. Crum RJ and Loiselle RJ, Oral perception and propioception: A review o the literature and its significance to prosthodontics. J Prosthet Dent, 1972. 28: p. 215-226.

27. Crum RJ, Loiselle RJ, and Hayes $\mathrm{CK}$, The stud attachment overlay denture and propioception. J Am Dent Assoc, 1971. 82: p. 583.

28. Pacer FJ and Bowman DC, Occlusal force discrimination by denture patients. J Prosthet Dent, 1975. 33: p. 602.

29. Morrow RM, et al., Tooth-supported complete dentures: an approach to preventive prosthodontics. J Prosthet Dent, 1969. 21: p. 513-22.

30. Lord JL and Teel S, The Overdenture. Dent Clin North Am, 1969. 13: p. 87181.

31. Ettinger RL, Taylor TD, Scandrett FR, Treatment needs for Overdenture patients in a longitudinal study: five year results. J Prosthet Dent, 1984. 52(4): p. $532-7$. 
32. Toolson LB, Taylor TD, A ten year report of a longitudinal recall of overdenture patients. J Prosthet Dent, 1989. 62: p. 179-81.

33. Mensor, M.C., Attachment fixation for overdentures. Part I. J Prosthet Dent, 1977. 37(4): p. 366-371.

34. Mensor MC, The rationale of resilient hinge-action stressbreakers. J Prosthet Dent, 1968. 20: p. 204-215.

35. Brewer AA and Morrow RM, Overdentures. 2nd ed. 1980, St. Louis: Mosby Company. 208-251.

36. Mensor MC, Classification and selection of attachments. J Prosthet Dent, 1977. 29: p. 494-497.

37. Fenner W, Gerber AA, and Muhlemann HR, Tooth mobility changes during treatment with partial denture prosthesis. J Prosthet Dent, 1956. 6: p. 520525.

38. Dalla-Bona H, Personal communication, Mensor MC, Editor. 1962.

39. Leung T and Preiskel HW, Retention Profiles of Stud-Type Precision Attachments. Int J Prosthodont, 1991. 4: p. 175-179.

40. Lehmann KM and Arnim FV, Studies on the retention forces on snap-on attachments. Quin Dent Technol, 1978. 7: p. 45.

41. Steward $\mathrm{BL}$ and Edwards $\mathrm{RO}$, Retention and wear of precision-type attachments. J Prosthet Dent, 1983. 49: p. 28-34.

42. Laird WRE and Grant AA, The use of magnetic forces in prosthetic dentistry. Int J Prosthodont, 1981. 4: p. 175-179. 
43. Dolder EJ, The bar joint mandibular denture. J Prosthet Dent, 1961. 11: p. 689-707.

44. Mensor MC, Attachment fixation for overdentures. Part I. J Prosthet Dent, 1977. 37(4): p. 366-371.

45. Davis DM, The shift in the therapeutic paradigm: Osseointegration. J Prosthet Dent, 1998. 79: p. 37-42.

46. Bodine RL, Evaluation of 27 mandibular subperiosteal implant dentures after 15 to 22 years. J Prosthet Dent, 1974. 32: p. 188--97.

47. Branemark PI, Zarb GA, and Albrektsson T, Tissue-integrated prostheses. Osseointegration in clinical dentistry. 1985, Chicago: Quintessense Books.

48. Schmitt A and Zarb GA, The notion of implant-supported overdentures. J Prosthet Dent, 1998. 79: p. 60-5.

49. Zarb GA and S. JM, Osseointegrated dental implants: preliminary report on a replication study. J Prosthet Dent, 1983. 50: p. 271-6.

50. Kent G, Effects of osseointegrated implants on psychological and social wellbeing: A literature review. J Prosthet Dent, 1992. 68: p. 515-518.

51. Carlsson GE, Otterland A, and Wennstrom A, Patient factors in appreciation of complete dentures. J Prosthet Dent, 1967. 17: p. 322-328.

52. Raghoebar GM, et al., Effectiveness of three implant modalities for the edentulous mandible. A five-year randomized clinical trial. Clin Oral Implants Res, 2000. 11: p. 195-201. 
53. Geertman ME, et al., Denture satisfaction in a comparative study of implantretained mandibular overdentures: A randomized clinical trial. Int J Oral Maxillofac Implants, 1996. 11: p. 194-200.

54. Feine JS, et al., Within-subject comparisons of implant-supported mandibular prostheses: choice of prosthesis. J Dent Res, 1994. 73: p. 1105-11.

55. Wismeijer D, et al., Patient satisfaction with implant-supported mandibular overdentures. A comparison of three treatment strategies with ITI-dental implants. Int J Oral Maxillofac Implants, 1997. 26: p. 263-7.

56. Sadowsky SJ, Mandibular implant-retained overdentures: A literature review. J Prosthet Dent, 2001. 86: p. 468-73.

57. Menicucci G, et al., Mandibular implant-retained overdenture: finite element analysis of two anchorage systems. Int J Oral Maxillofac Implants, 1998. 13: p. 369-76.

58. Naert I, et al., Prosthetic aspects of osseointegrated fixtures supporting overdentures. A 4 year report. J Prosthet Dent, 1991. 65: p. 671-80.

59. Cune MS, et al., Treatment outcome with implant-retained overdentures: Part II. Patient satisfaction and predictability of the subjective treatment outcome. J Prosthet Dent, 1994. 72: p. 152-8.

60. Krennmair $\mathrm{G}$ and Ulm $\mathrm{C}$, The symphyseal single-tooth implant for anchorage of a mandibular complete denture in geriatric patients: a clinical report. Int J Oral Maxillofac Implants, 2001. 16: p. 98-104.

61. Mericske-Stern RD and Zarb GA, Clinical protocol for treatment with implant-supported overdentures. 11th ed. Boucher's prosthodontic treatment 
for edentulous patients, ed. Zarb GA, Bolender CL, and Carlsson GE. 1997, St. Louis: Mosby.

62. Mish CE, Contemporary Implant Dentistry. 2nd ed. 1999, St Louis: Mosby. 179.

63. Burns DR, Mandibular Implant Overdenture Treatment: Consensus and Controversy. J Prosthod, 2000. 9: p. 37-46.

64. Watson AJ, Tinsley D, and Sharma S, Implant complications and failures. The complete overdenture. Dent Update, 2001. 28: p. 234-240.

65. Gulizio MP, Agar JR, Kelly JR, et al., Effect of implant angulation upon retention of overdenture attachments. J Prosthodont, 2005. 14: p. 3-11.

66. Weimeyer AS, Agar JR, and Kazemi RB, Orientation of retentive matrices in spherical attachments independent of implant parallelism. J Prosthet Dent, 2001. 86: p. 434-7.

67. Walton JN, A randomized clinical trial comparing two mandibular implant overdenture designs: 3 year prosthetic outcomes using a six-field protocol. Int J Prosthodont, 2003. 16: p. 255-260.

68. Watson GK, et al., Mandibular overdentures: comparative evaluation of prosthodontic maintenanec of three different implant systems during the fist year of service. Int J Prosthodont, 2002. 15: p. 259-266.

69. Walton JN, MacEntee MI, and Glick N, One year prosthetic outcomes with implant overdentures: A randomized clinical trial. Int J Oral Maxillofac Implants, 2002. 17: p. 391-398. 
70. Gotfredsen $\mathrm{K}$ and Holm B, Implant-supported mandibular overdentures retained with a ball or bar attachments: a randomized prospective 5-year study. Int J Prosthodont, 2000. 13: p. 125-130.

71. Porter JA, Petropoulos VC, and B. JB, Comparison of the Load Distribution for Implant Overdenture Attachments. Int J Oral Maxillofac Implants, 2002. 17: p. 651-662.

72. Thompson GW and Kreisel PS, The impact of demographics of aging and the edentulous condition on dental care services. J Prosthet Dent, 1998. 79: p. 5659.

73. Walton JN and R. ND, In vitro changes in clips and bars used to retain implant overdentures. J Prosthet Dent, 1995. 74: p. 482-6.

74. Gamborena JI, et al., Retention of ERA direct overdenture attachments before and after fatigue loading. Int J Prosthodont, 1997. 10: p. 123-130.

75. Setz J, Lee SH, and Engel E, Retention of prefabricated attachments for implant stabilized overdentures in the edentulous mandible: An in vitro study. J Prosthet Dent, 1998. 80: p. 323-9.

76. Chung KH, Chung CY, and Cagna DR, Retention characteristics of attachment systems for implant overdentures. J Prosthodont, 2004. 13: p. 221226.

77. Petropoulos VC and Smith W, Maximum dislodging forces of implant overdenture stud attachments. Int J Oral Maxillofac Implants, 2002. 17: p. 526-535. 
78. Van Kampen F, et al., Retention and Postinsertion maintenanace of bar-clip, ball and magnet attachments in mandibular implant overdenture treatment: and in vivo comparison after 3 months of function. Clin Oral Impl Res, 2003. 14: p. 720-726.

79. Sarnat AE, The efficiency of cobalt samarium magnets as retention units for overdentures. J Prosthet Dent, 1983. 11(4): p. 324-333.

80. Botega DM, et al., Retention force and fatigue strenght of overdenture attachment systems. J Oral Rehab, 2004. 31: p. 884-889.

81. Landa LS, et al., A prospective 2-year clinical evaluation of overdentures attached to nonsplinted implants using ERA attachments. Pract Proced Aesthet Dent, 2001. 13(2): p. 151-156.

82. Albrektsson T, et al., The long term effcacy of currently used implants: $A$ review and proposed criteria of success. Int J Oral Maxillofac Implants, 1986. 1: p. 11-24.

83. Straumann, Straumann ITI. Techno Info. 1997: Waldenburg, Switzerland.

84. Walmsely A and Frame J, Implant supported overdentures-the Birmingham experience. J Dent Res, 1997. 25 (suppl): p. 45-7.

85. Zarb GA and Schmitt A, The edentulos predicament, II: the longitudinal effectiveness of implant supported overdentures. J Am Dent Assoc, 1996. 127: p. 66-72.

86. Ortegon SM, Agar JR, and Thompson J, The use and misuse of ball attachments in mandibular overdentures, in Poster (Greater New York 
Academy of Prosthodontics). 2004, University of Connecticut Health Center:

New York.

87. Rutkunas V and Mizutani H, Retentive and Stabilizing Properties of Stud and Magnetic Attachments Retaining Mandibular Overdenture. An in vitro study. Stomatologia, Baltic Dental and Maxillofacial Journal, 2004. 6(3): p. 85-90.

88. Gulizio MP, Effect of withdrawal path upon retention of spherical retentive anchors, in Graduate Prosthodontics. 2003, University of Connecticut: Farmington, CT. p. 34-35.

89. Anusavice KJ, Phillips' science of dental materials. 10th edition ed, ed. Anusavice KJ. 1996, Philadelphia, PA,: WB Saunders. 254-55.

90. Naert I, et al., A 5 year prospective randomized clinical trial on the influence of splinted and unsplinted oral implants retaining a mandibular overdenture: prosthetic aspects and patient satisfaction. J Oral Rehab, 1999(26): p. 195202.

91. Watson RM, et al., Prosthodontic treatment, patient response, and the need for maintenance of complete implant-supported overdentures: an appraisal of 5 years of prospective study. Int J Prosthodont, 1997. 10: p. 345-54.

92. Davis DM and Packer ME, Mandibular overdentures stabilized by Astra Implants with either ball or magnets: 5 year results. Int J Prosthodont, 1999. 12: p. 222-9. 


\section{List of Tables}

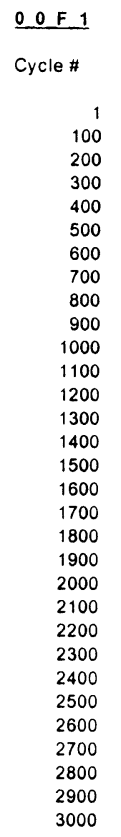

\begin{tabular}{rr}
\multicolumn{1}{l}{ Peak } & \multicolumn{1}{l}{ Valley } \\
& \\
27.317 & -38.83 \\
18.78 & -27.072 \\
17.912 & -26.974 \\
17.969 & -27.496 \\
17.394 & -27.336 \\
17.515 & -27.778 \\
17.703 & -27.092 \\
17.391 & -27.712 \\
17.468 & -27.722 \\
17.517 & -26.613 \\
17.431 & -26.081 \\
17.566 & -26.22 \\
17.653 & -27.63 \\
17.695 & -27.263 \\
17.744 & -27.817 \\
17.274 & -27.523 \\
17.567 & -27.846 \\
17.617 & -26.502 \\
17.769 & -26.595 \\
17.543 & -27.011 \\
17.209 & -26.625 \\
17.561 & -26.96 \\
17.41 & -25.773 \\
17.525 & -26.457 \\
17.509 & -26.146 \\
17.662 & -27.228 \\
17.71 & -26.48 \\
17.708 & -27.182 \\
17.856 & -27.282 \\
17.611 & -26.7 \\
18.012 & -27.594 \\
\hline &
\end{tabular}

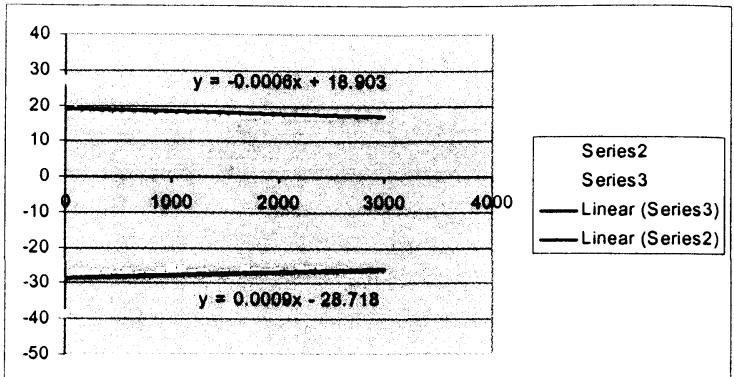

\begin{tabular}{lrr} 
Mean Peak Load & 17.658 & \\
Mean Valley Load & -27.252 & \\
SD Peak Load & 0.471 & \\
SD Valley Load & 0.747 & \\
& & \\
& & \\
& & \\
\hline & 17.874 & -26.45 \\
0 & 17.758 & -27.009 \\
\hline & 17.853 & -27.23 \\
& 17.943 & -26.641 \\
& 18.197 & -27.987
\end{tabular}

Table 1. Table shows one of the 60 Excel spread-sheets used to tabulate the MTS data. (0-0 Specimen $1 \mathrm{~F}$ ) 


\section{List of Figures}

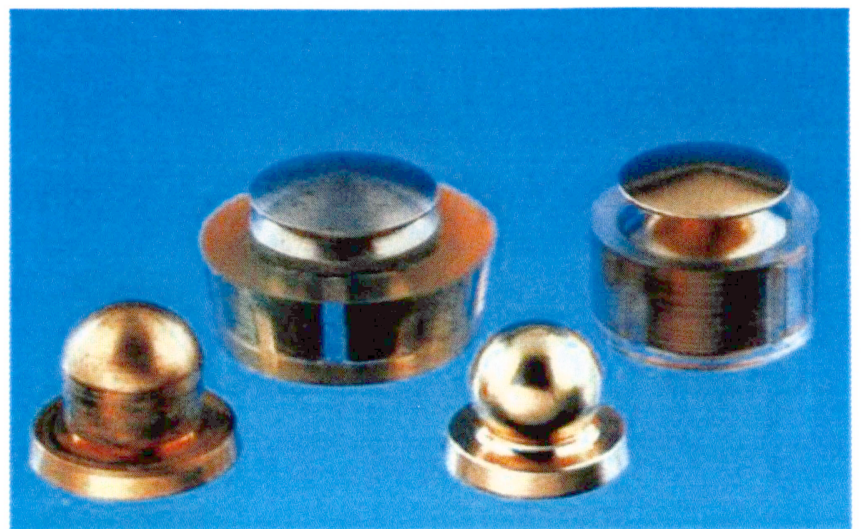

Figure 1. Dalbo series: gold lamella and housing. PVC sleeve. No rotational capability over the abutment. Preiskel H, Overdentures made easy.

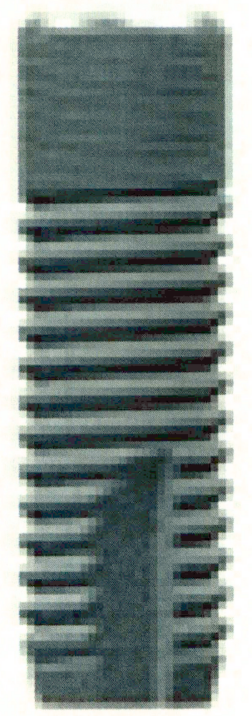

Figure 2. Astra Implant $4.0 \times 13 \mathrm{~mm}$ 


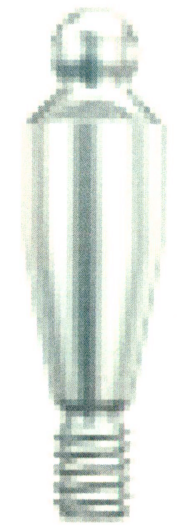

Figure 3. 2.25 Diameter, 1.5 height Astra Ball Abutment

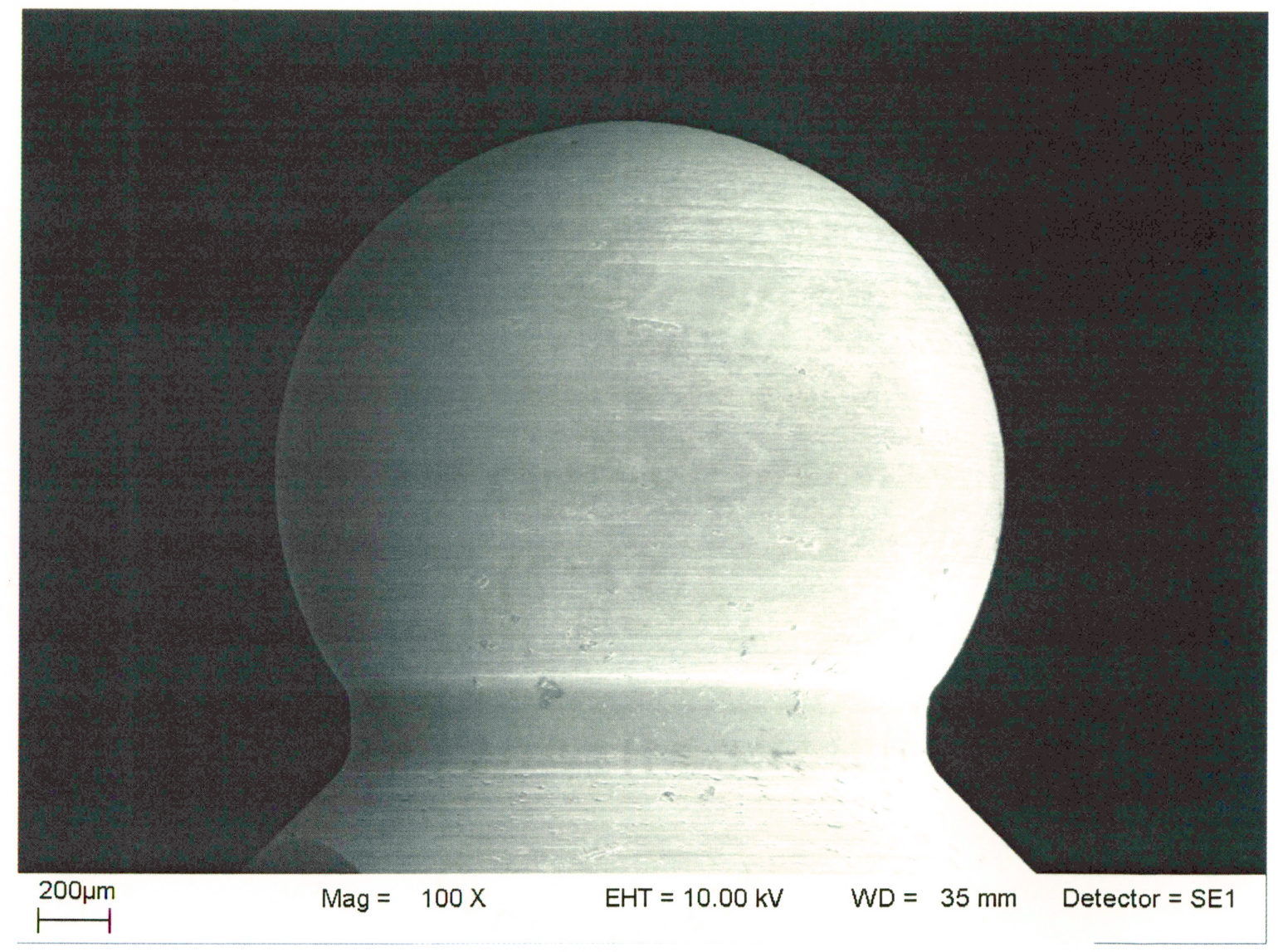

Figure 4. New $2.25 \mathrm{~mm}$ spherical abutment (SEM) 


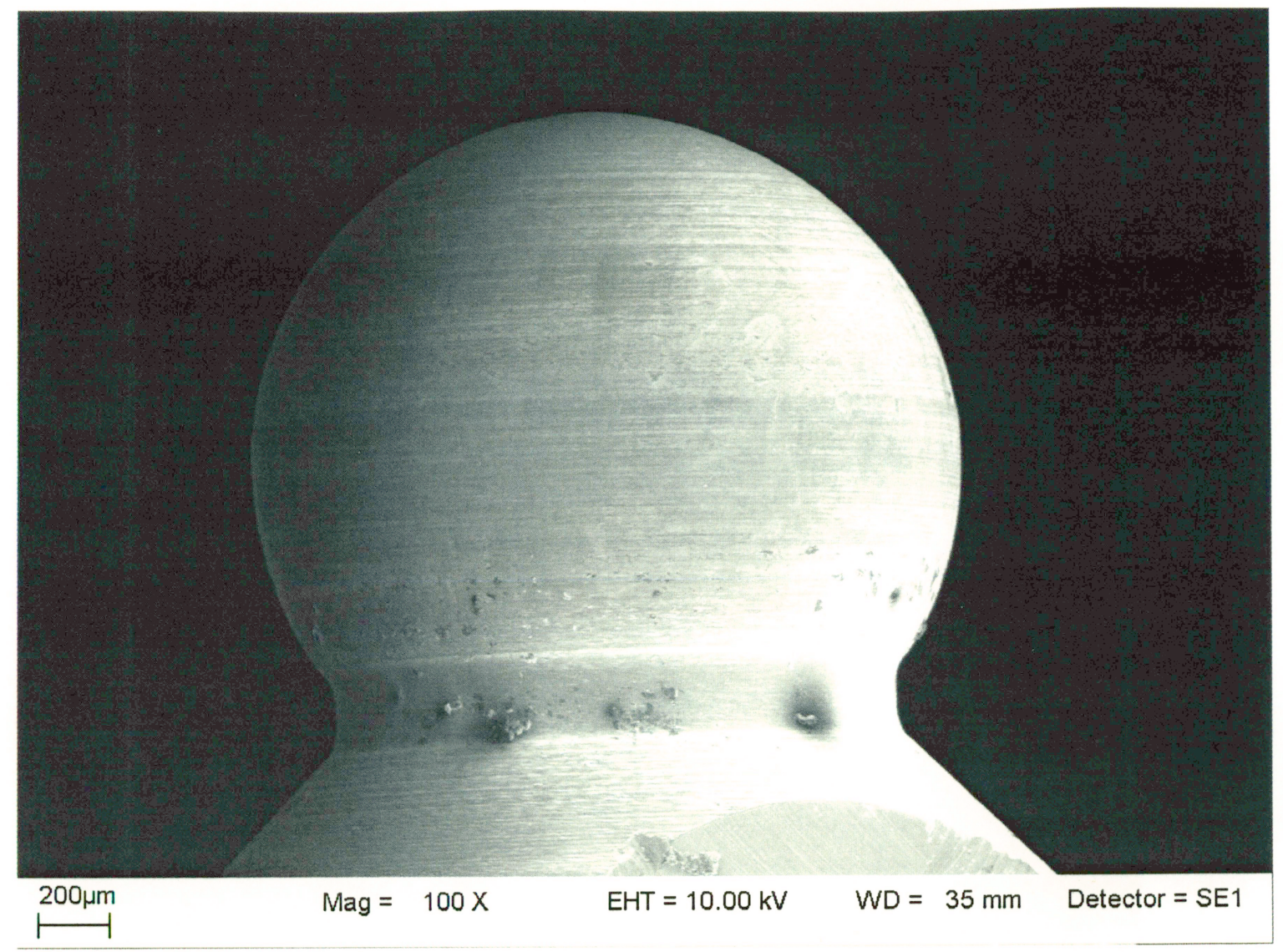

Figure 5. Figure depicts a ball abutment after cycling, note the vertical striations

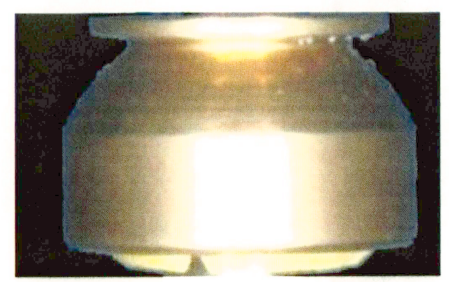

Figure 6. Preci-Clix Female Attachment (Housing and Insert)

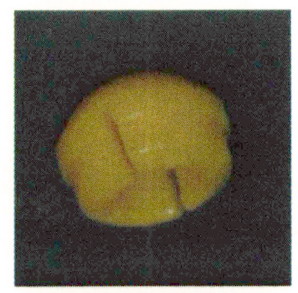

Figure 7. Plastic Insert 


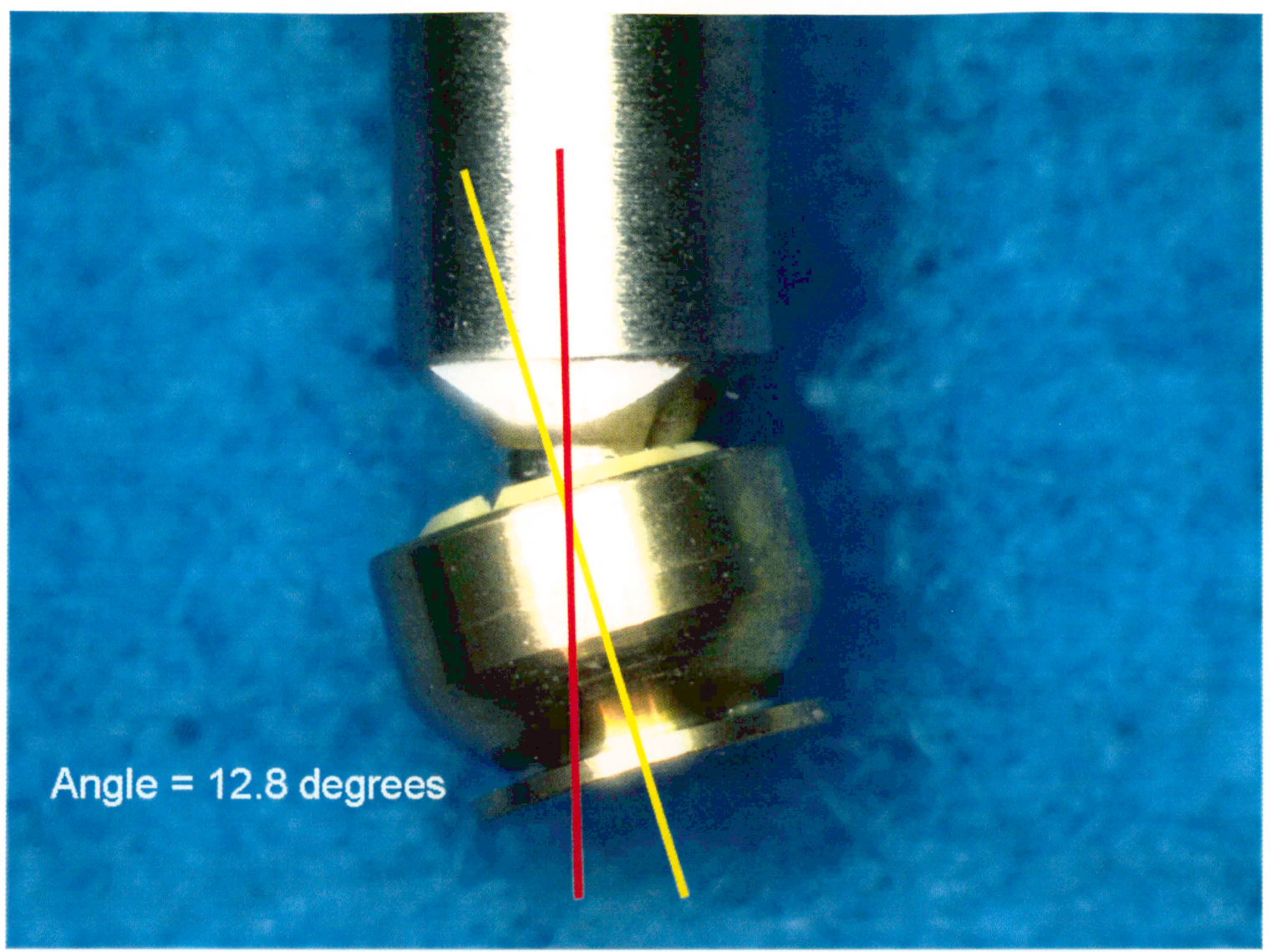

Figure 8. Rotational Capability Threshold of Preci-Clix attachment on an Astra implant. 12.8 degrees 


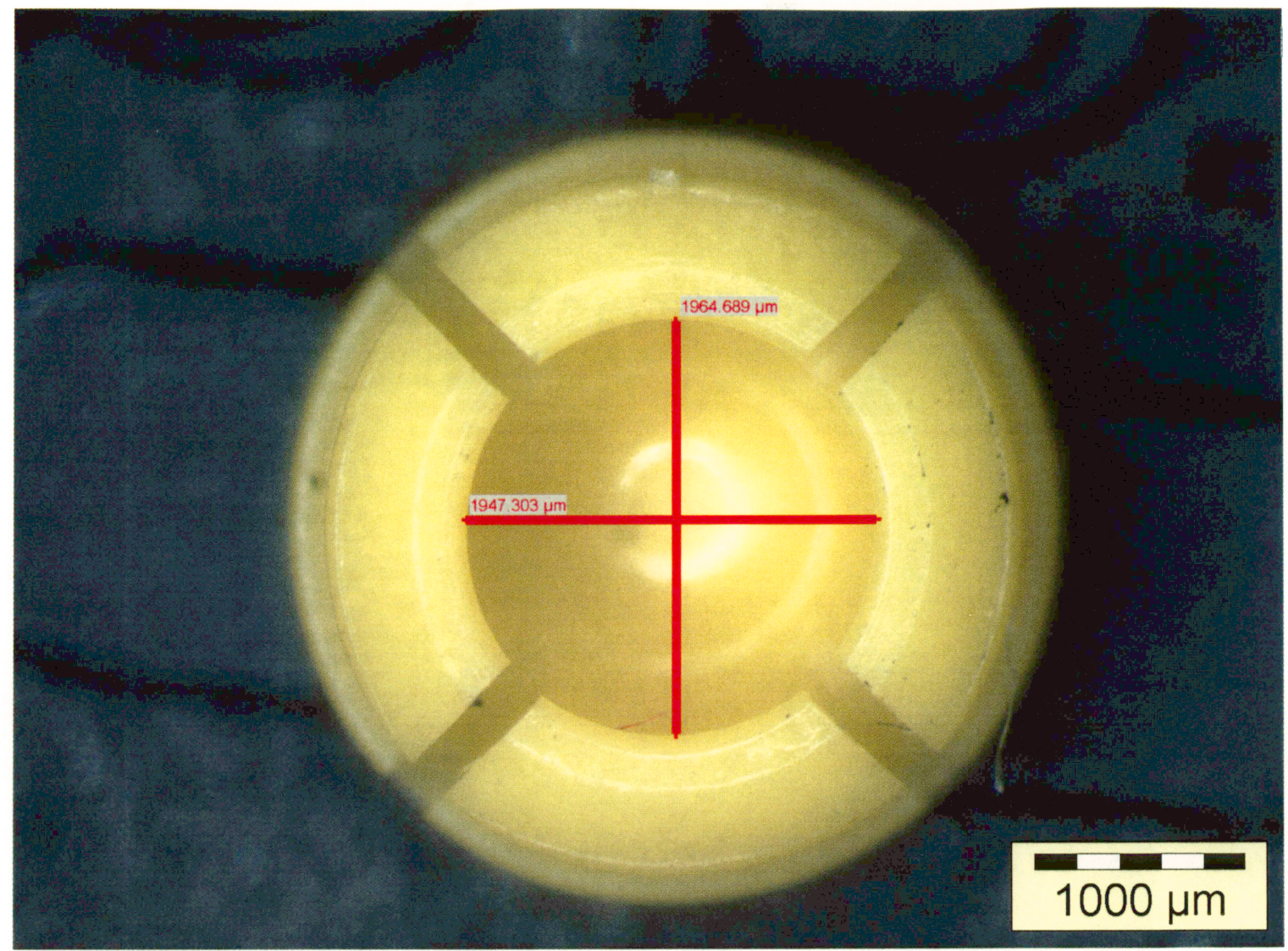

Figure 9. Diameter of a new plastic insert 


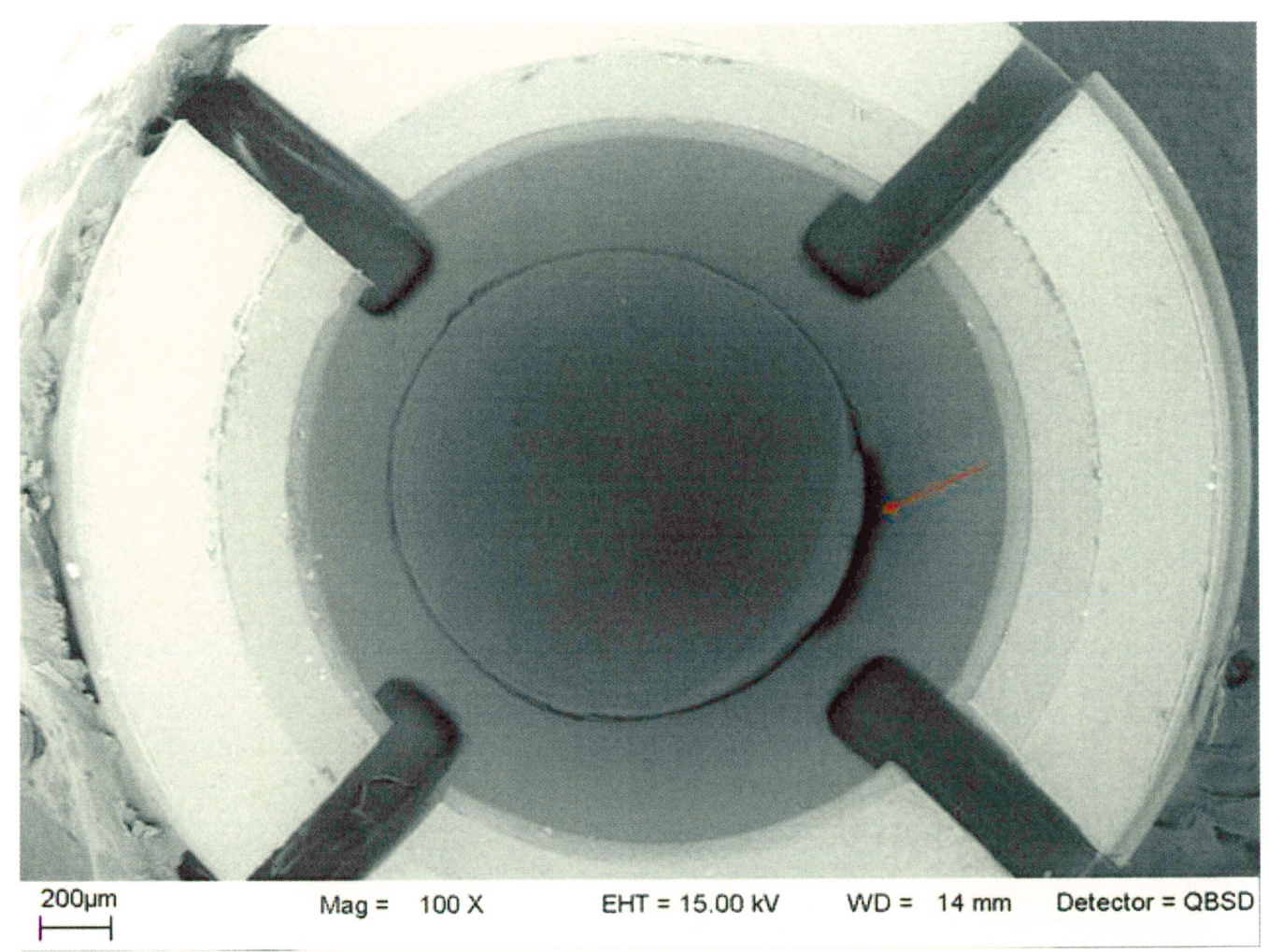

Figure 10. SEM views of randomly selected new attachments showed fabrication discrepancies 


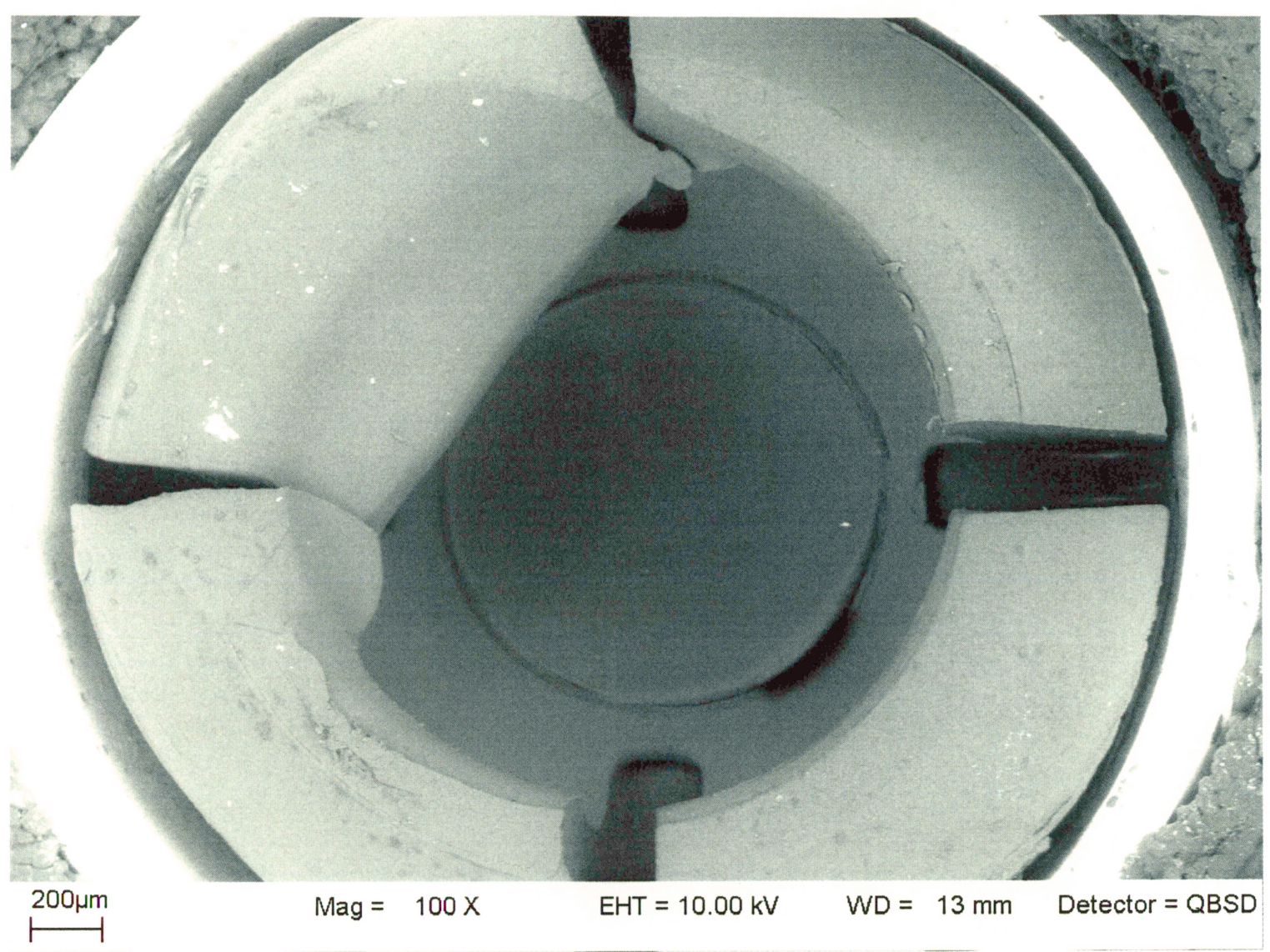

Figure 11. Figure depicts a SEM image of an attachment tested on the 5th group (15-15) 


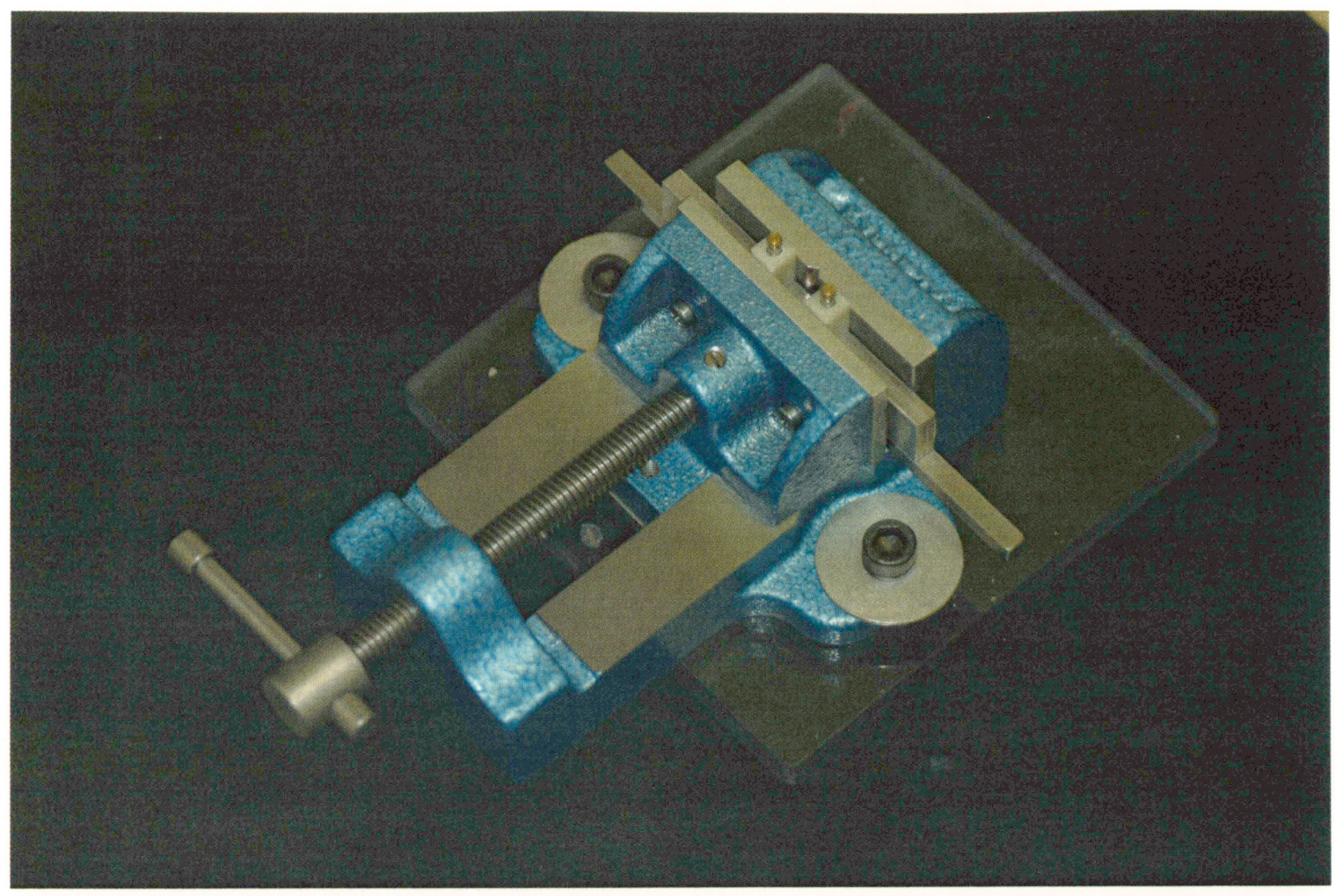

Figure 12. Professional Vise (Mandible Analog)

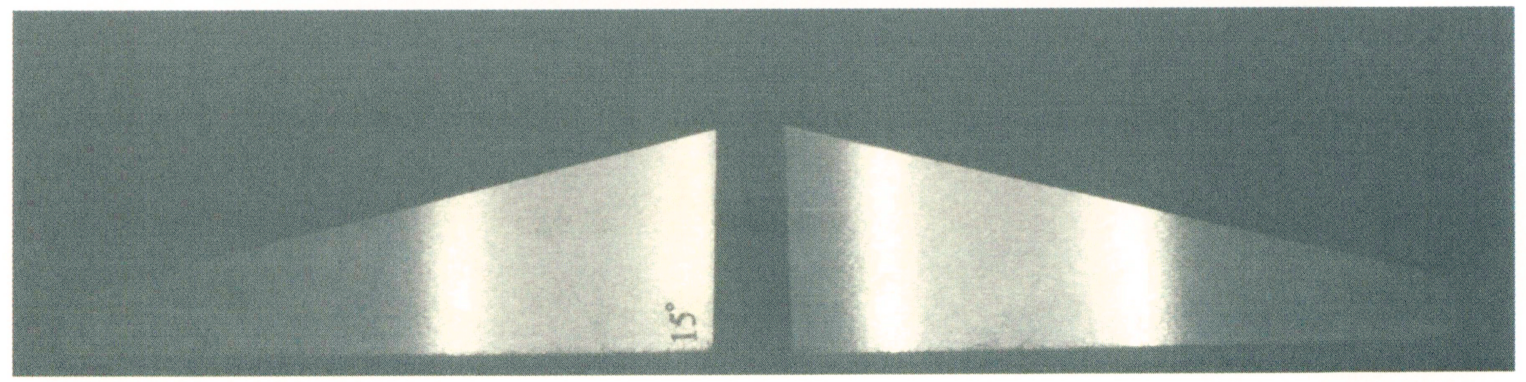

Figure 13. Aluminum Angled Blocks (15 ${ }^{\circ}$ pair) 


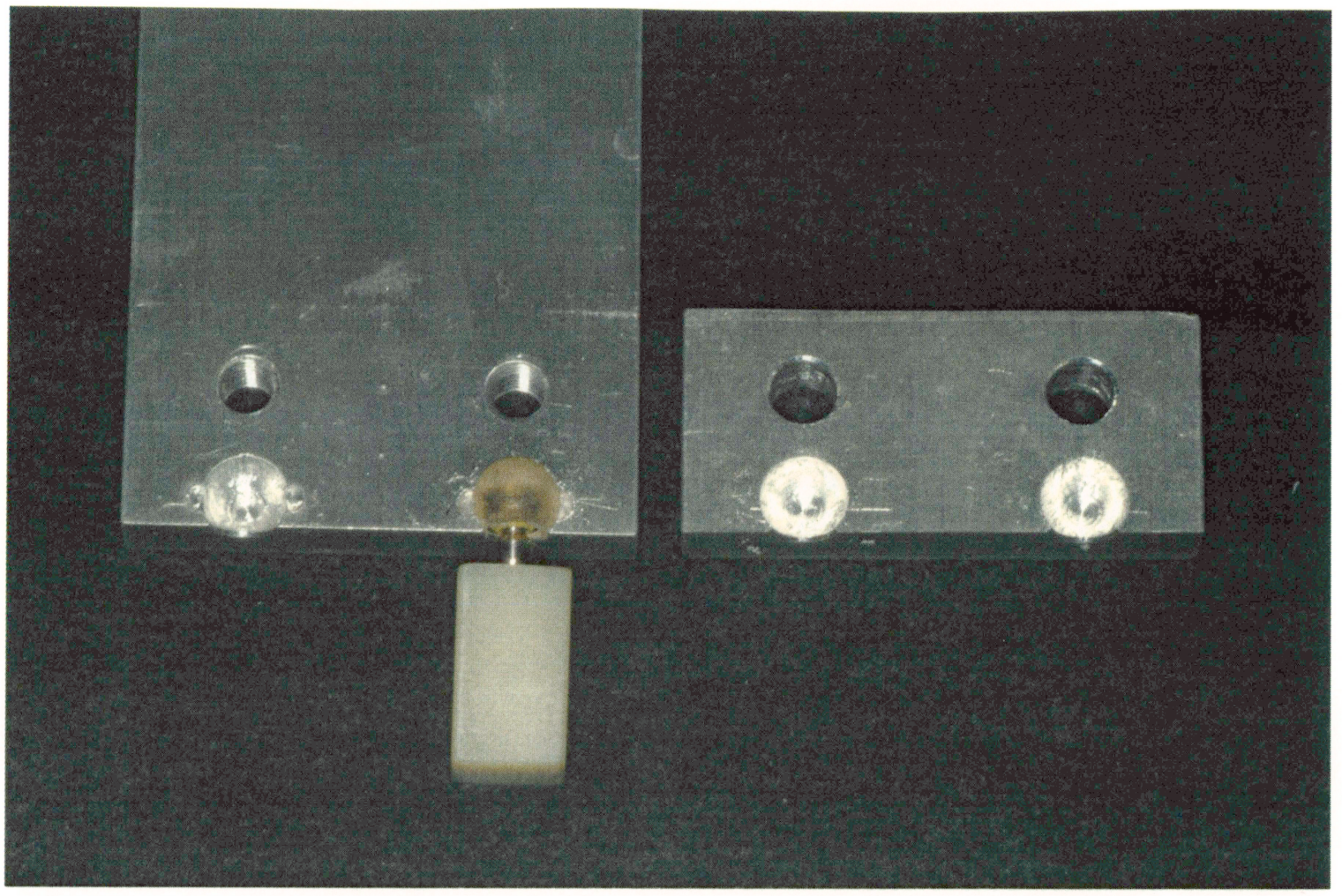

Figure 14. Upper Aluminum Tool 


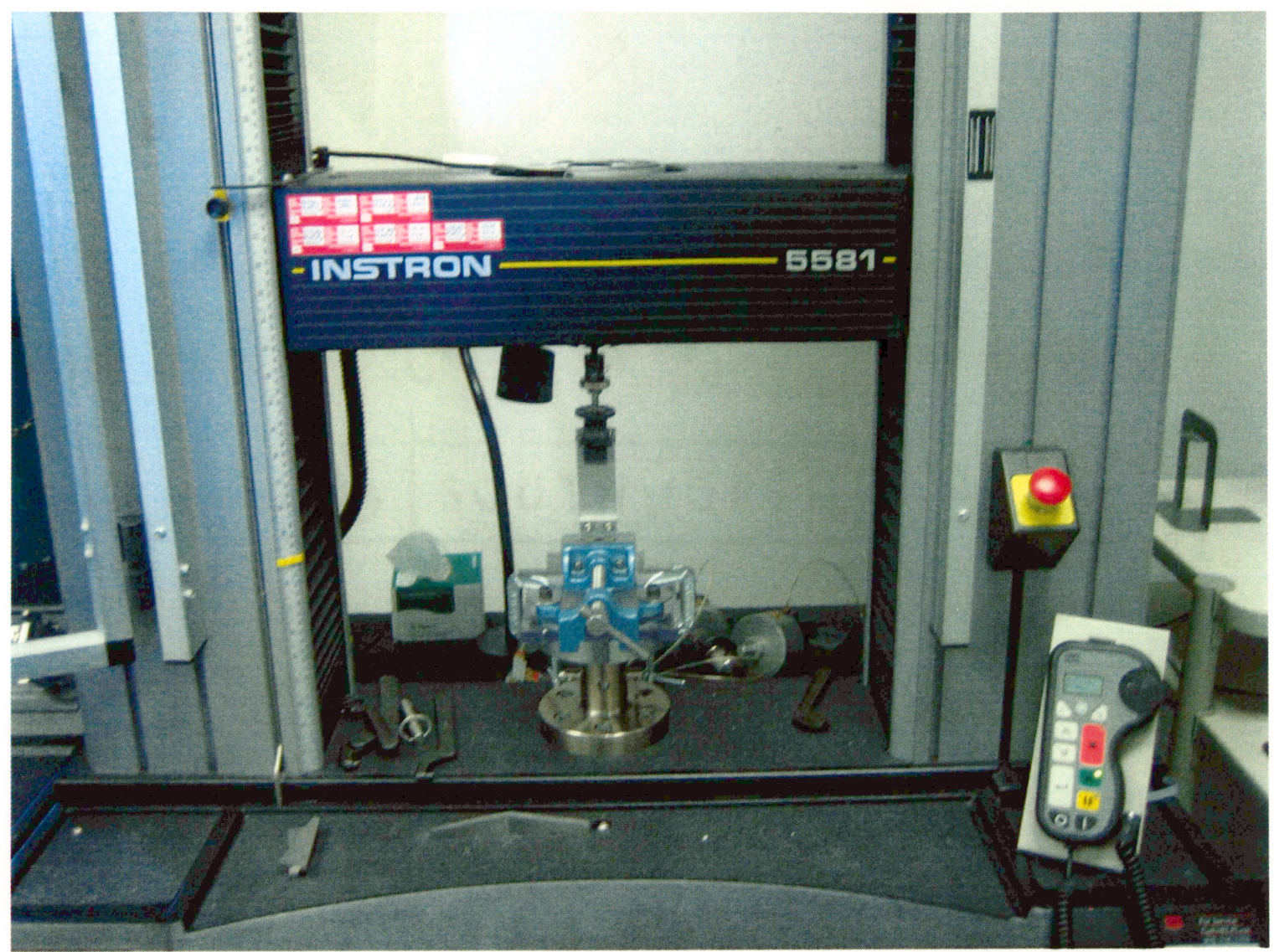

Figure 15. Instron 5581 series, Norwood, MA 


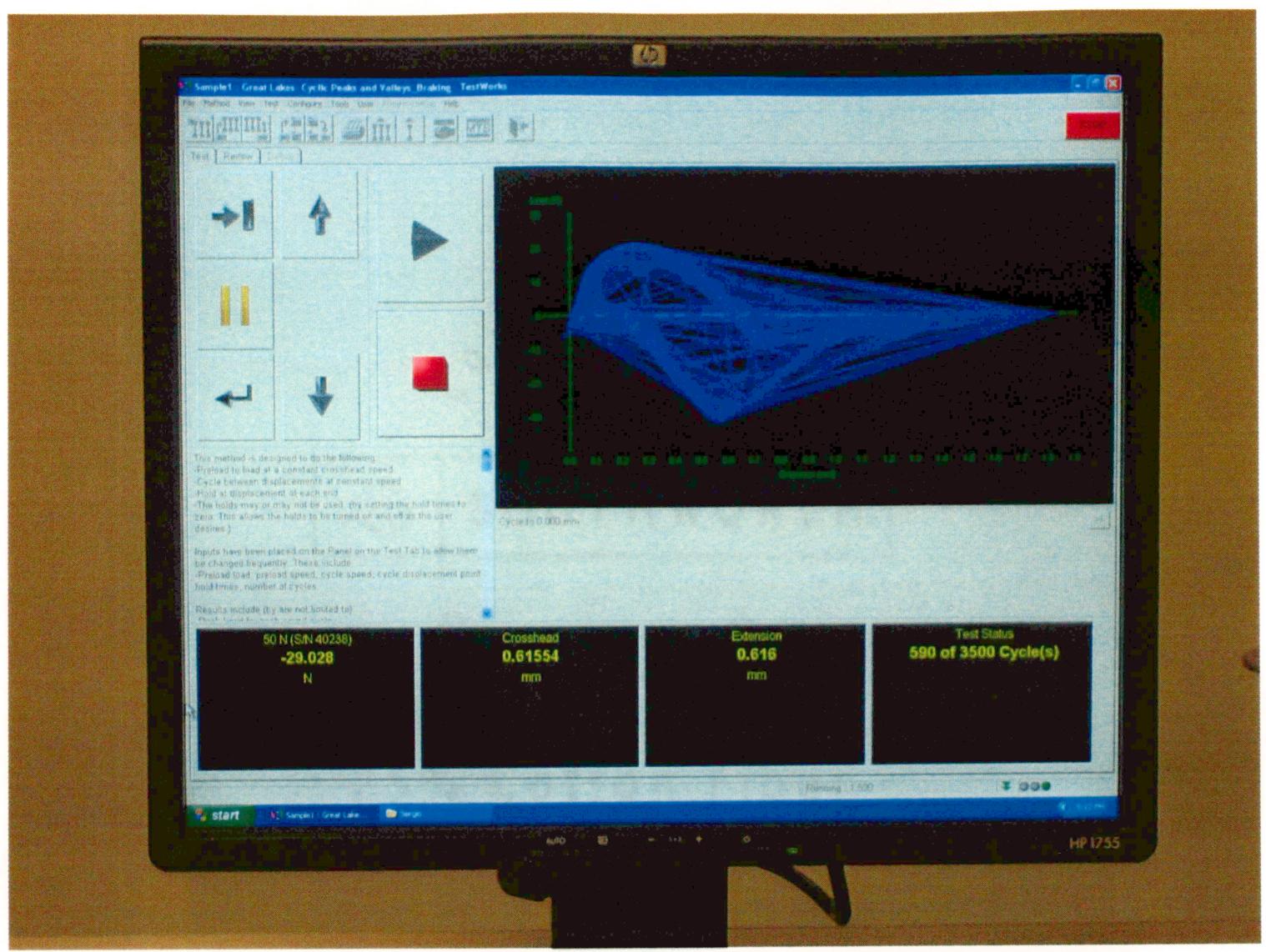

Figure 16. Test Works 4®, Version 4.08 B Build 868 Copyright $(\subset)$ 1995-2003 MTS systems corporation 
1) $0-0$

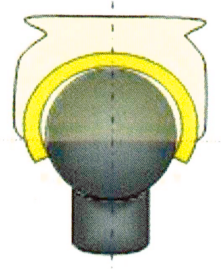

3) $15-0$
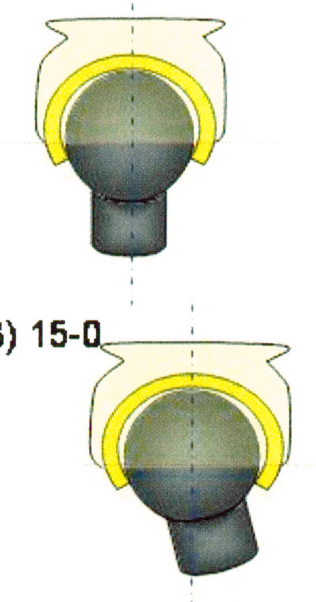

4) $10-10$

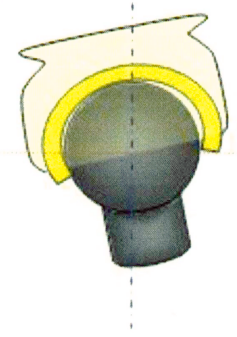

2) $10-0$
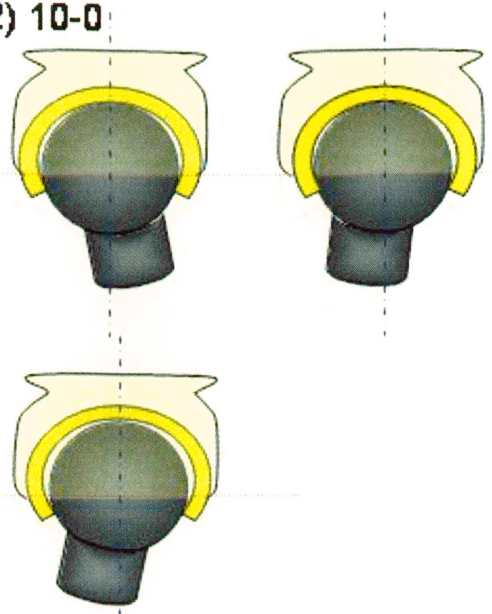

5) $15-15$

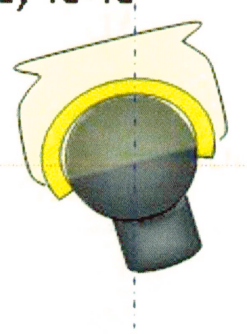

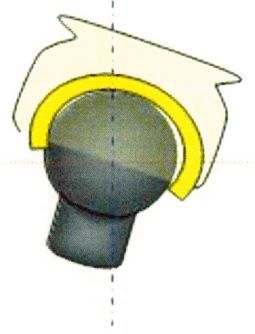

Figure 17. Implant-Attachment Configurations:

1) $0^{\circ}$ implants- $0^{\circ}$ attachments $\left.(\mathrm{N}=6) ; 2\right) 10^{\circ}$ implants- $0^{\circ}$ attachments $(\mathrm{N}=6)$; 3) $15^{\circ}$ implants- $0^{\circ}$ attachments $(\mathrm{N}=6)$; 4) $10^{\circ}$ implants- $10^{\circ}$ attachments $\left.(\mathrm{N}=6) ; 5\right) 15^{\circ}$ implants- $15^{\circ}$ attachments $(\mathrm{N}=6)$. 


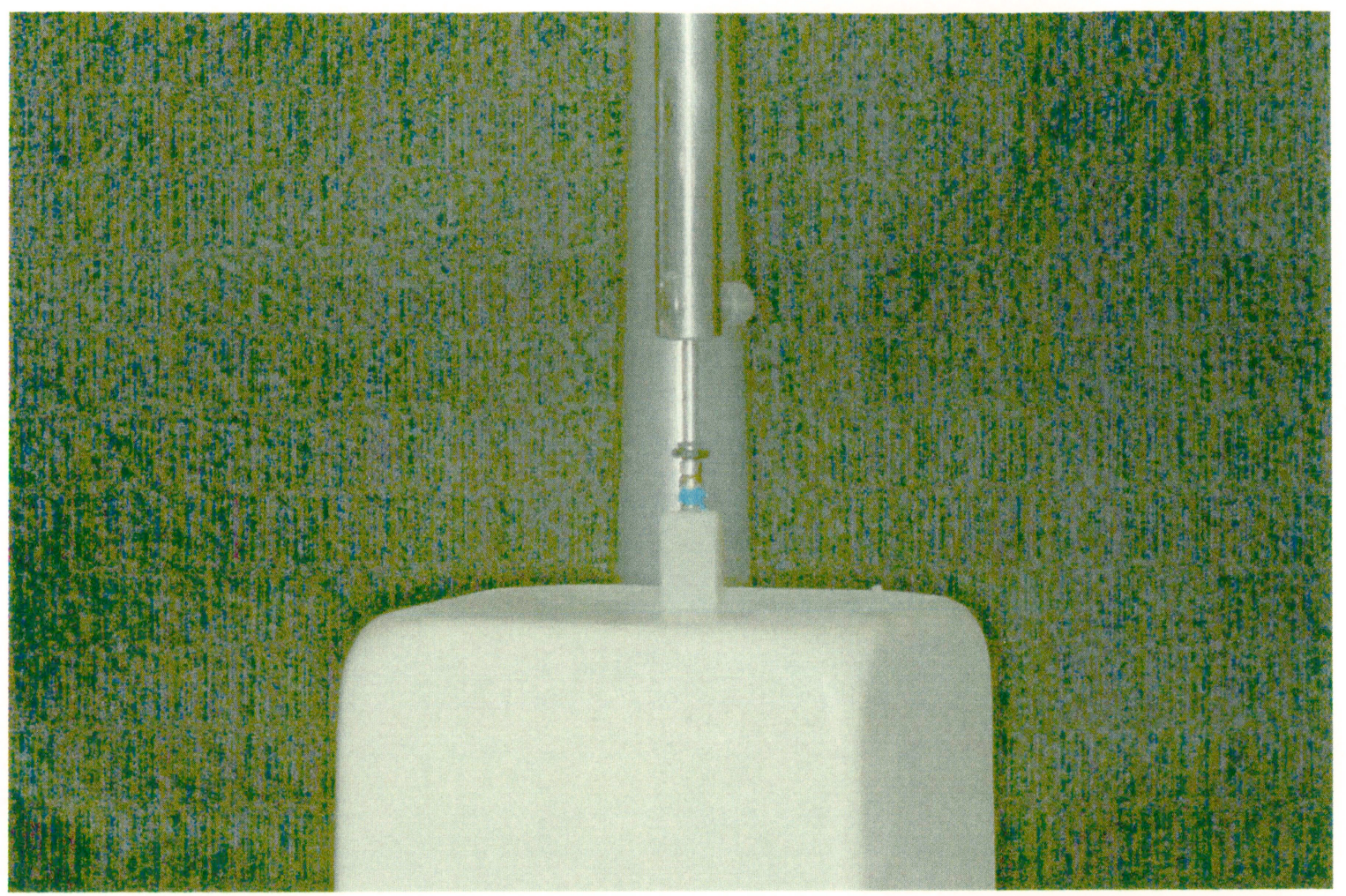

Figure 18. Attachment Insertion and Fixation prior processing

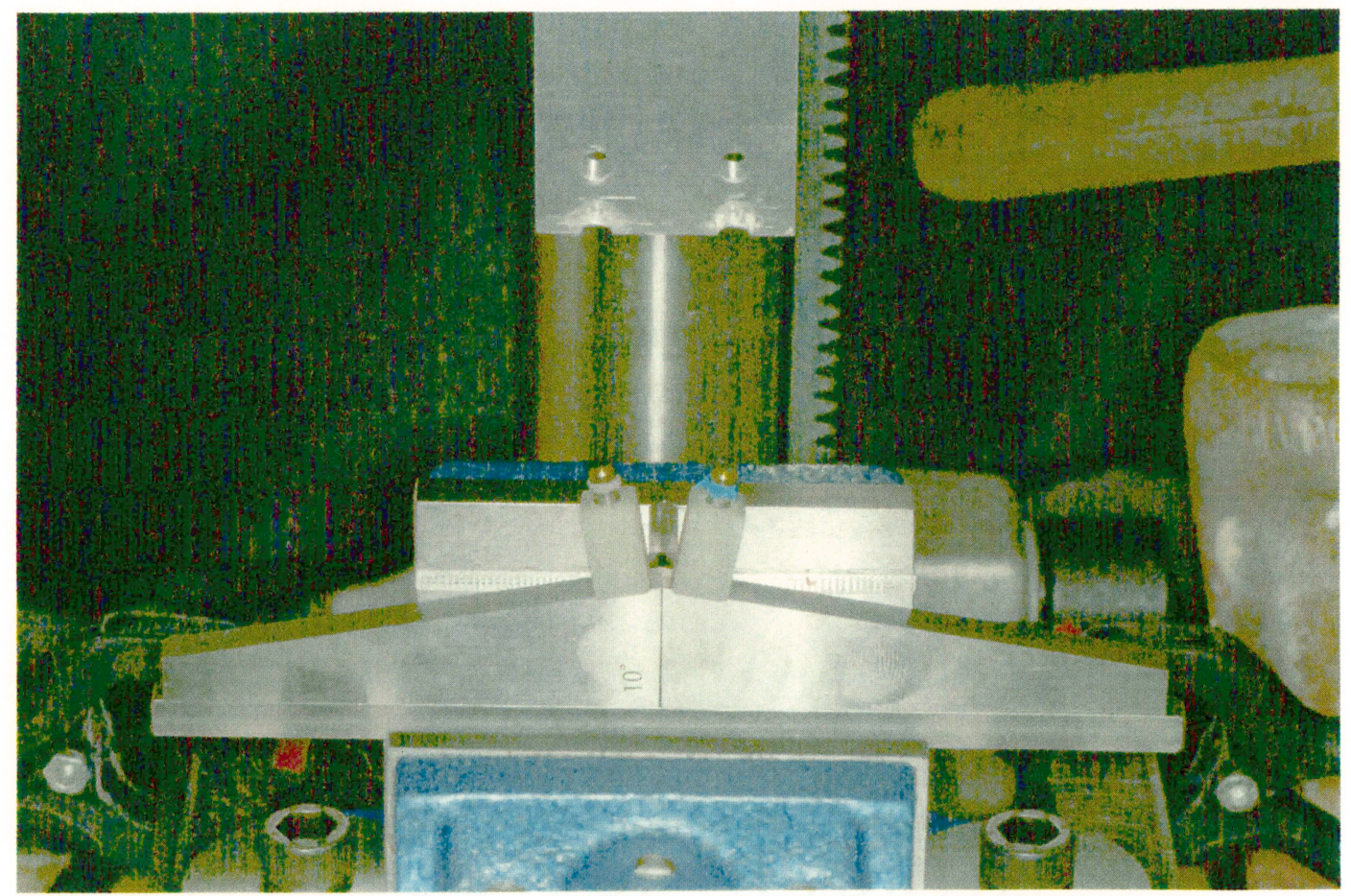

Figure 19. Aluminum blocks being used on a vise to direct angulation of the abutments and attachments which in turn run perpendicular to their slope. This figure shows a partially opened vise with 10 degree Aluminum blocks 


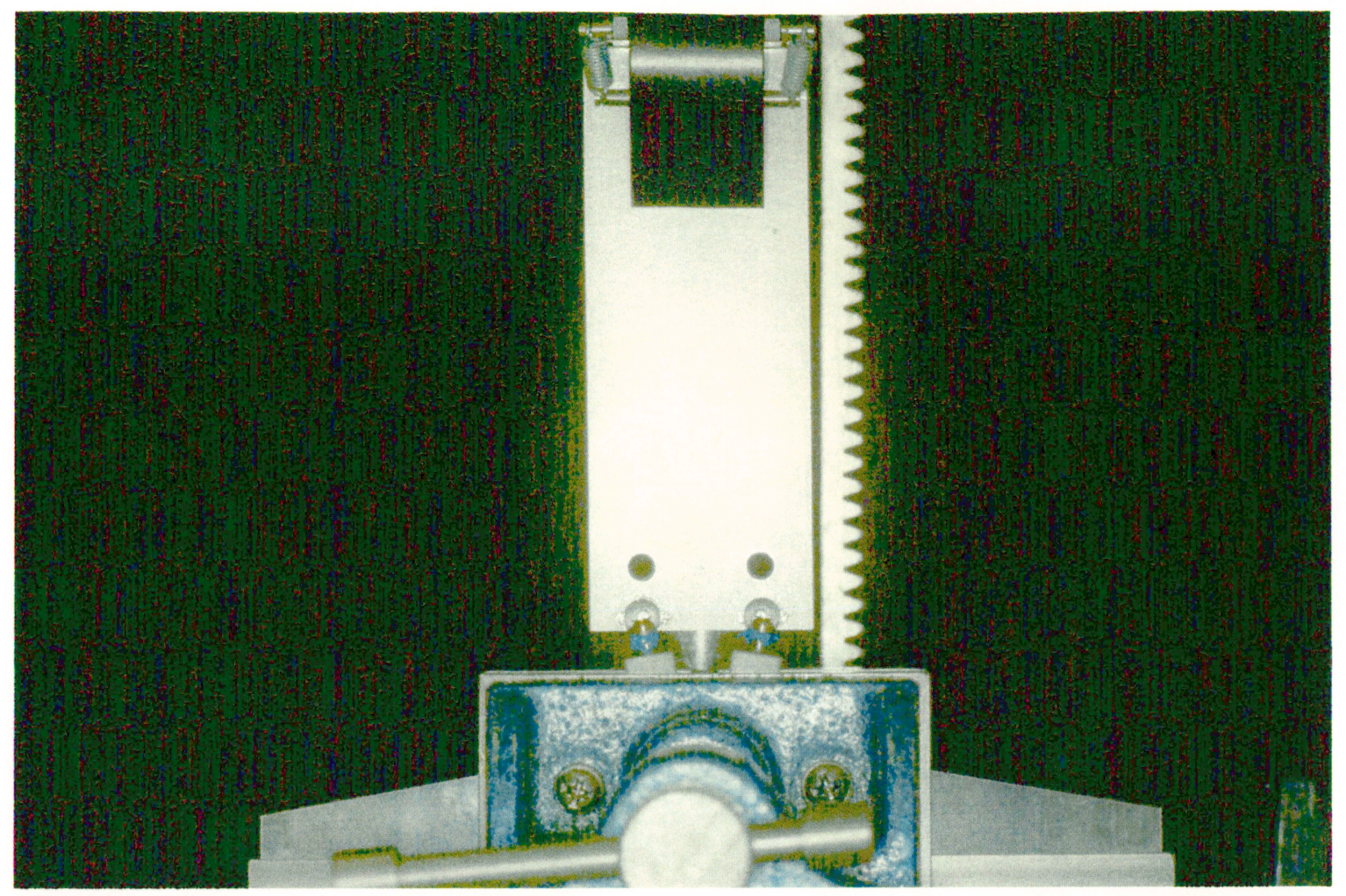

Figure 20. Attachment processing. Figure shows the Upper Aluminum tool being held into position prior injecting the acrylic resin. 


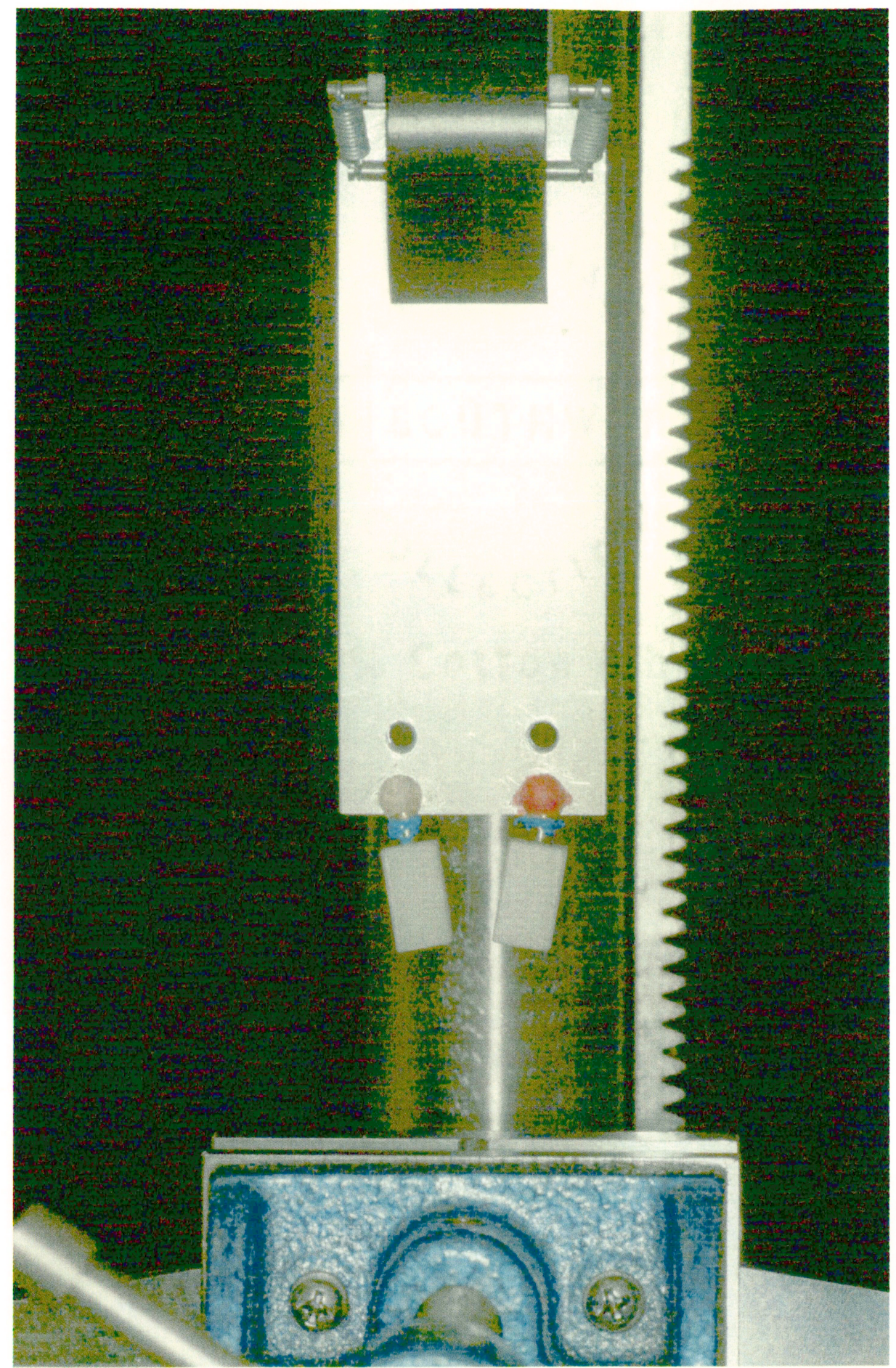

Figure 21. Attchment Processing. Figure depicts the appearance of the acrylic resin after setting and removing the top half of the Upper Aluminum tool. Grip has been raised. 


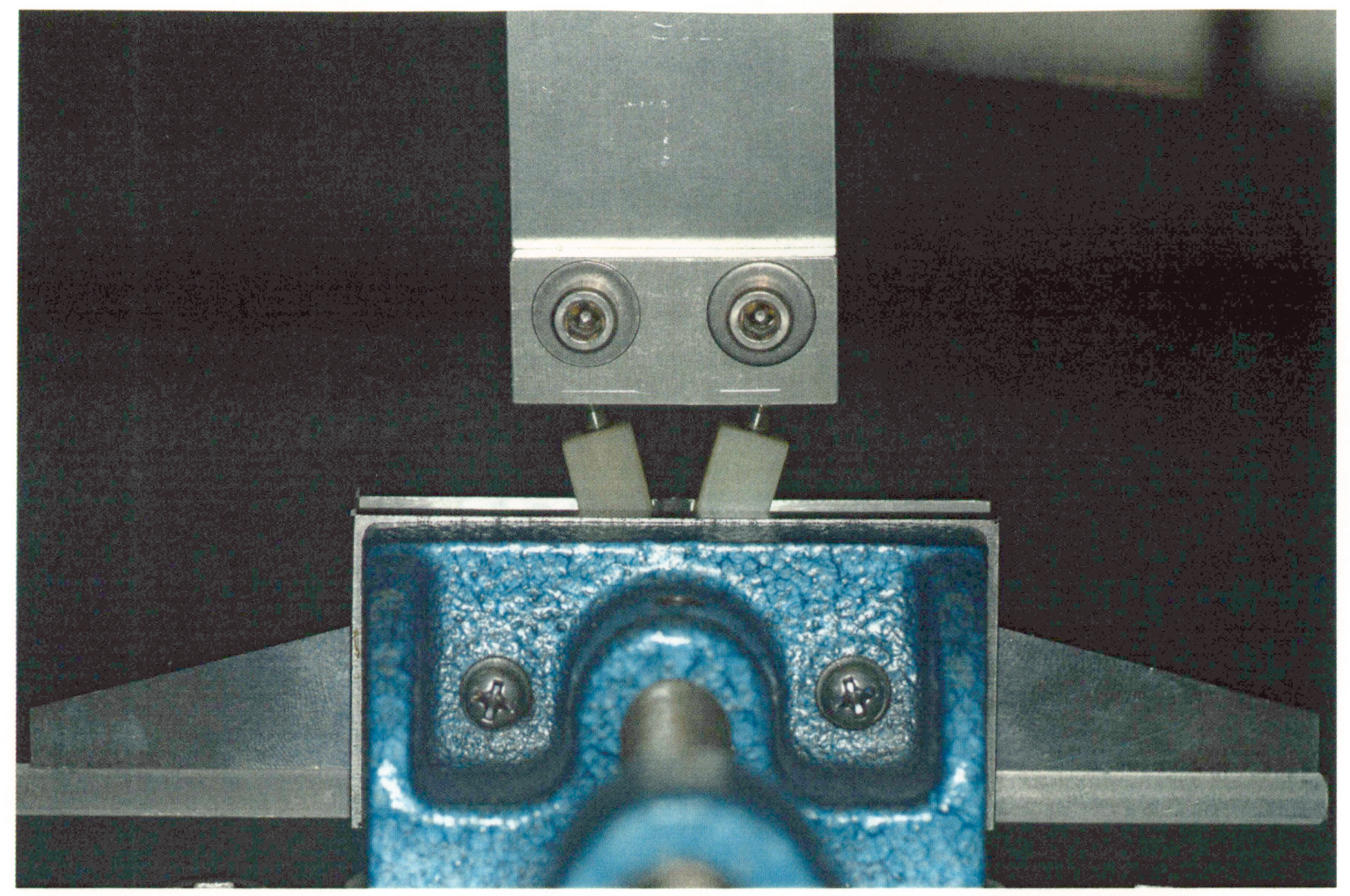

Figure 22. Upper and Lower tools denoting the 0 degree attachment insertion to 15 degree implants 


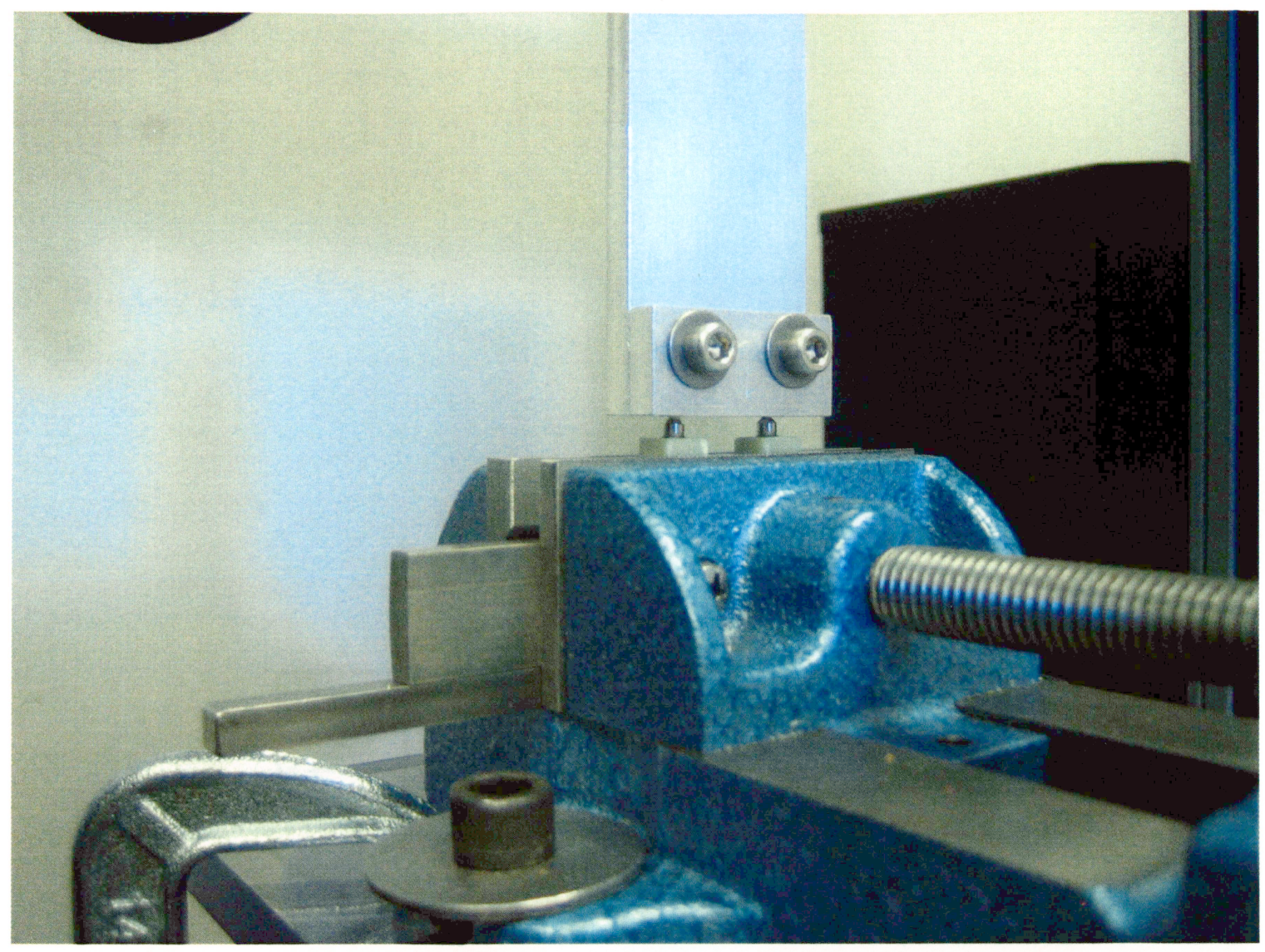

Figure 23. Upper and Lower tools holding parallel implants and parallel attachments 


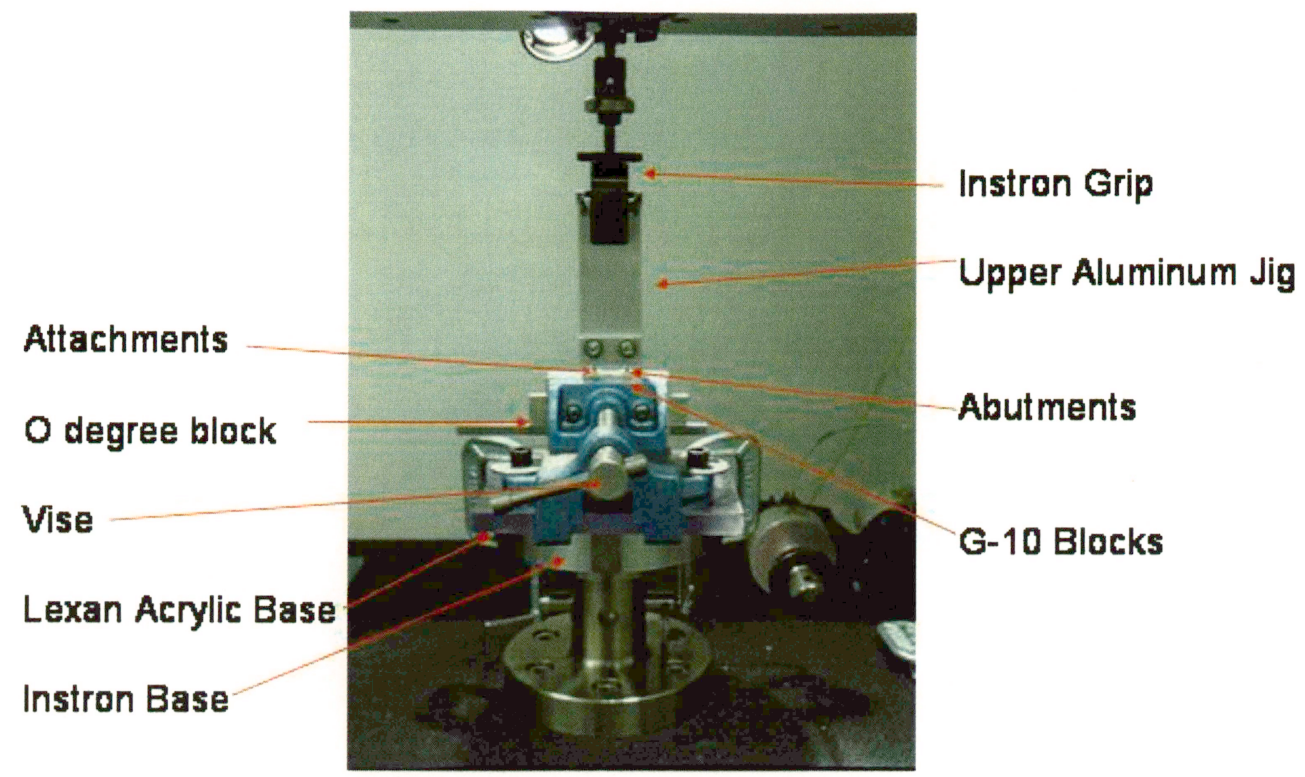

Figure 24. Complete setup on Instron Machine

4) $10-10$

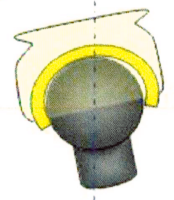

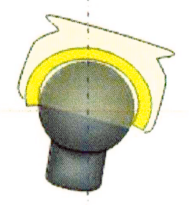

5) $15-15$

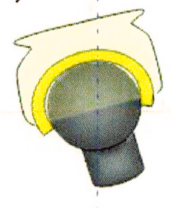

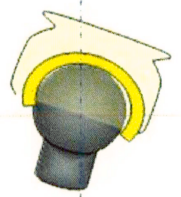

Figure 25. Configurations 4 and 5, Attachments processed following non-parallel implants long axis 


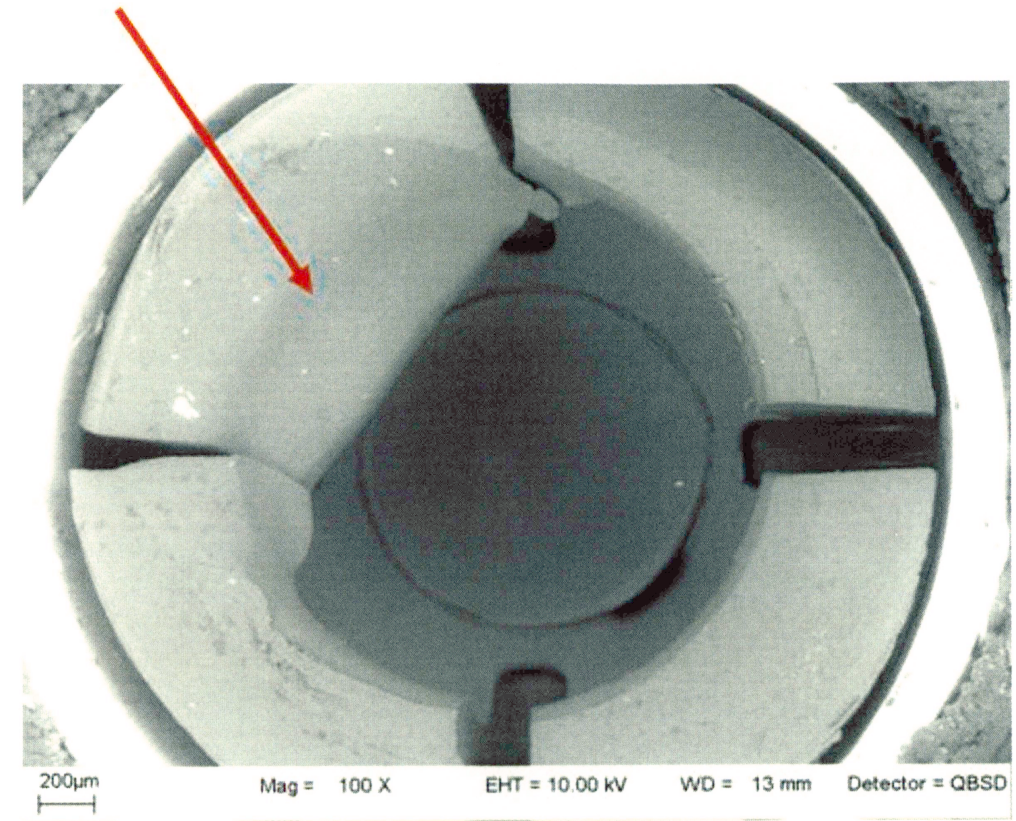

Figure 26. Arrow depicts plastic permanent deformation on the 15-15 sample (5th group) 


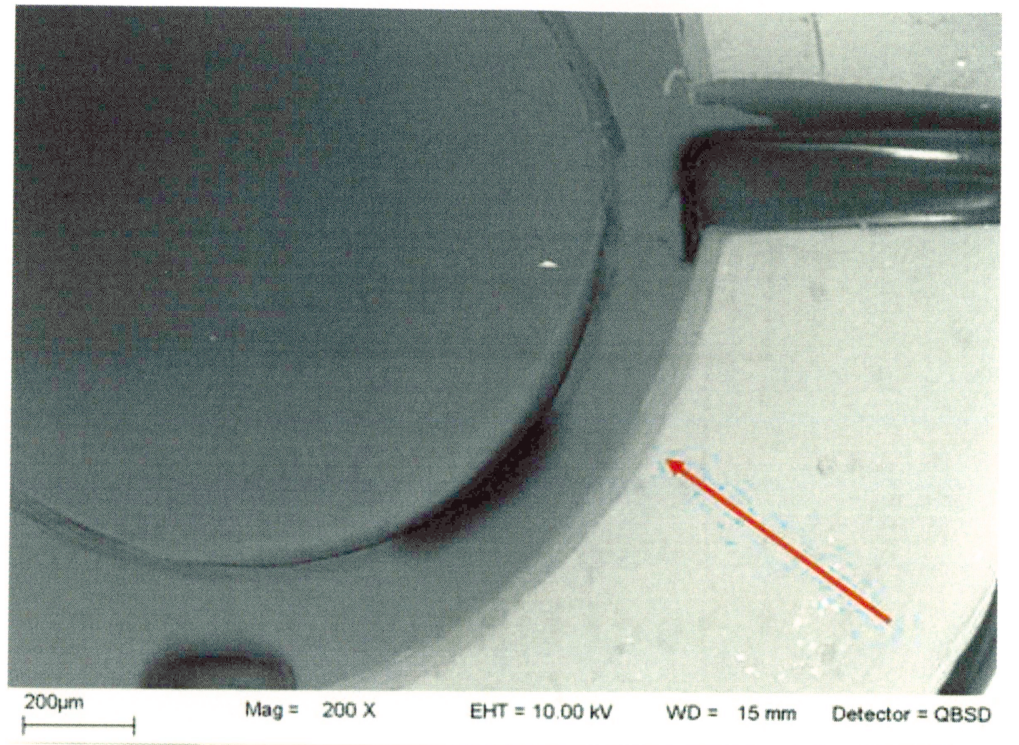

Figure 27. Arrow depicts the contraleteral side of one cylced 15-15 plastic insert. Note the lack of changes 


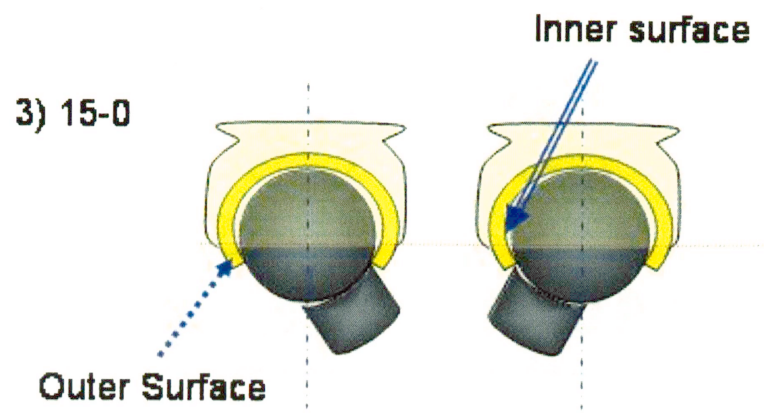

Figure 28. Diagram depicts the 15-0 configuration, internal and external aspects are labeled 


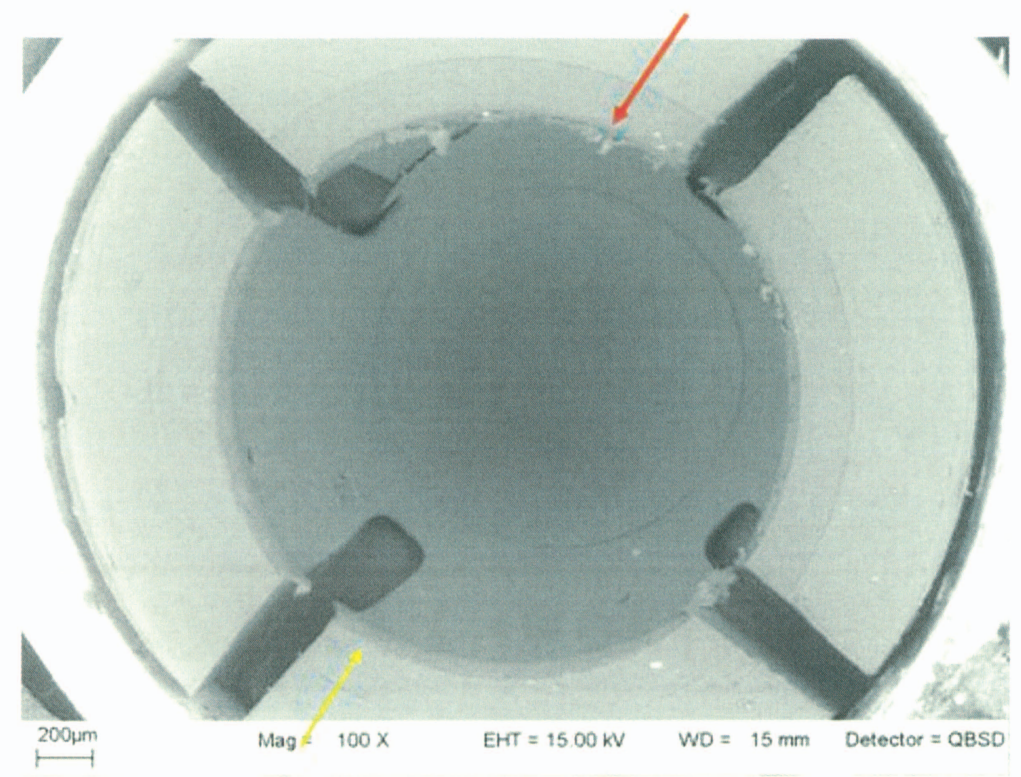

Figure 29. Red arrow depicts internal aspect, yellow indicates external. See difference in wear patterns 


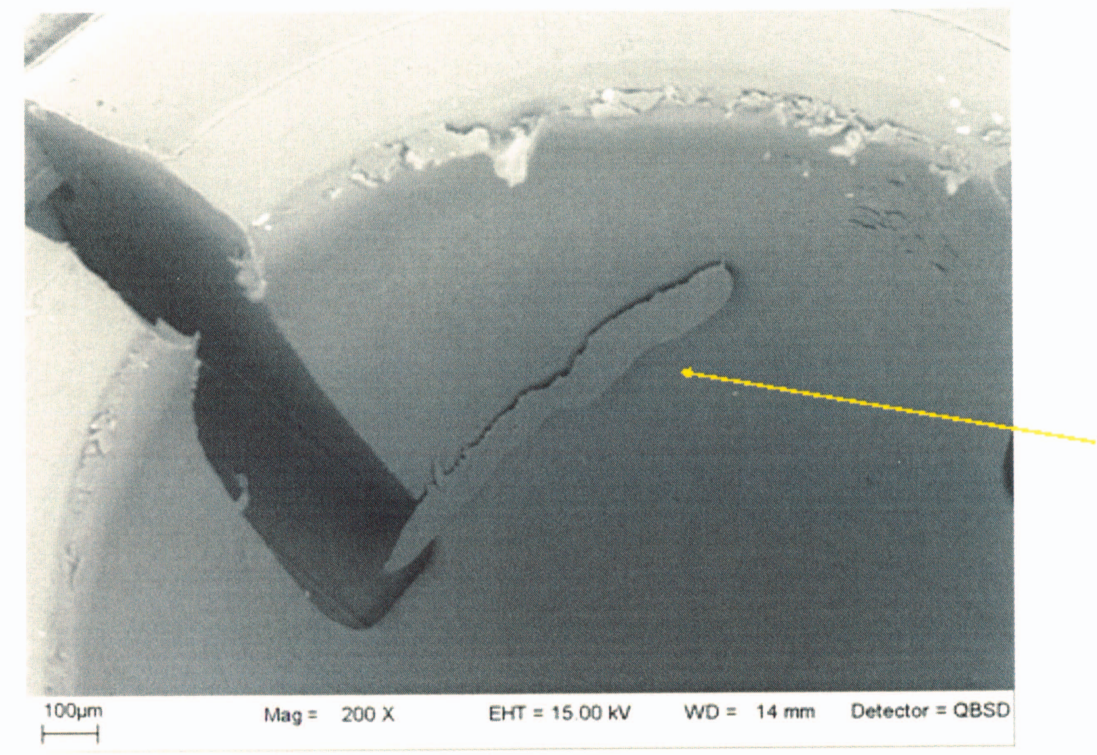

Figure 30. Arrow depicts plastic wear and debris on the 15-0 configuration 
